\title{
Antibiotics in the Pipeline for Treatment of Infections due to Gram-Negative Organisms
}

\author{
Nicole C. Vissichelli, $M D^{*}$ \\ Michael P. Stevens, MD, MPH
}

\author{
Address \\ "Division of Infectious Diseases, Department of Internal Medicine, Virginia Com- \\ monwealth University, 1250 E Marshall Street, PO Box 980509, Richmond, VA, \\ 23298-0509, USA \\ Email: Nicole.Vissichelli@vcuhealth.org
}

Published online: 8 May 2019

(C) Springer Science+Business Media, LLC, part of Springer Nature 2019

This article is part of the Topical Collection on Antimicrobial Stewardship

Keywords Gram-negative · Antibiotic development · Antimicrobial resistance

\begin{abstract}
Purpose of review We systematically review the literature on antibiotics approved by the Food and Drug Administration since 2015 or that are in development through phase III trials for the treatment of infections due to gram-negative organisms.

Recent findings The crisis of antimicrobial resistance creates a dire need for the development of novel antimicrobials with enhanced activity against resistant bacteria. Of the agents that are currently in the pipeline or recently approved, few are from new classes and most represent modifications of preexisting agents. There continues to be a lack of treatment options especially for infections caused by organisms that produce metallo- $\beta$ lactamases. Furthermore, there is a paucity of data investigating the efficacy of these new antimicrobials for treatment of infections due to multidrug-resistant organisms.

Summary This review outlines the antimicrobials in development for the treatment of infections due to gram-negative bacteria. There are various agents in development that seem promising, but more studies are needed to evaluate the efficacy of these agents in treating infections, especially those due to multidrug-resistant organisms. The development of new antimicrobials is critical to combat the crisis of antimicrobial resistance.
\end{abstract}




\section{Introduction}

The development of antibiotics revolutionized the treatment of infectious diseases in the first half of the twentieth century. However, the subsequent emergence of antimicrobial resistance has raised the specter of a return to the pre-antibiotic era for many patients. Serious infections due to gram-negative organisms have been increasingly difficult to manage with the emergence of resistance via extended spectrum $\beta$-lactamases (ESBL) and the rise of carbapenem-resistant Enterobacteriaceae (CRE) and multidrug-resistant (MDR) organisms. There have been several initiatives calling for new antimicrobial development, including the Infectious Diseases Society of America's $10 \times$ '20 campaign in 2010, followed by the Generating Antibiotic Incentives Now (GAIN) act in the USA and the Innovative Medicines Initiative New Drugs for Bad Bugs by the European Union [1]. Both the World Health Organization and the United States Centers for Disease Control global report described antimicrobial resistance as a global health emergency requiring an urgent need for the development for alternative antibacterial agents $[2,3]$. Subsequently, there are various new antimicrobial agents in the pipeline for the treatment of gram-negative infections.

In this review, we summarize the available data for agents that were approved by the Food and Drug Administration (FDA) since 2015 or that are undergoing phase III clinical trials for the treatment of gram-negative infections.

\section{Methods}

Search strategy

A PubMed search was performed to identify clinical studies from January 1, 2000, through December 1, 2018 published in the English language. Antimicrobial agents that were FDA approved or undergoing phase III clinical trials from January 2015 through June 2018 were selected through the Pew Charitable Trust, FDA, and clinicaltrials.gov websites. These included cefiderocol, ceftazidime/avibactam (CZA), ceftobiprole medocaril, ceftolozane/ tazobactam $(\mathrm{C} / \mathrm{T})$, delafloxacin, eravacycline, relebactam (REL) in combination with imipenem/cilastatin (IMI), lefamulin, meropenem-vaborbactam (M-V), plazomicin, and sulopenem. PubMed search included each antimicrobial in combination with MESH terms, publication types, and topics to encompass all types of clinical trials, randomized controlled trials, meta-analysis, and multicenter, longitudinal, follow-up, prospective, retrospective, cohort, case-control, comparative, and/or observational studies. This was supplemented by review of references and clinicaltrials.gov to identify additional articles.

We included studies that focused on the clinical use of the selected antimicrobial agents for the treatment of gram-negative infections. Only primary studies were included. In vitro studies, animal studies, and pharmacokinetics (PK) were not systemically reviewed but pertinent current knowledge was also summarized. Only studies with infections due to gram-negative organisms were included.

\section{Results}

\section{Literature search and study selection}

Our literature search yielded 301 articles, of which 55 met inclusion criteria. The process of study selection is described in Fig. 1. 




Fig. 1. Articles included in the review.

Cefiderocol (S-649266) is a novel siderophore cephalosporin with a catechol side chain (Table 1). This allows ferric iron ion binding with a resulting complex that is transported into the bacteria and destroys cell wall synthesis [4]. It was studied at a dose of $2 \mathrm{~g}$ intravenously (IV) over $1 \mathrm{~h}$ every $8 \mathrm{~h}$. It is predominantly renally excreted and therefore requires renal dose adjustment [4]. It has no significant drug interaction potential via drug transporters in vivo [5]. It is stable against hydrolysis from all $\beta$-lactamases including serine Klebsiella pneumoniae carbapenemases (KPC), oxacillinase (OXA), and metallo- $\beta$ lactamases (MBL), including New Delhi MBL (NDM), imipenemase (IMP), and Verona integron-encoded MBL (VIM). It has potent activity against gramnegative organisms including CRE and MDR Pseudomonas aeruginosa, Acinetobacter baumannii, and Stenotrophomomans maltophilia in vitro [6-8].

A double-blind phase II randomized controlled trial (RCT) by Portsmouth et al. determined cefiderocol to be noninferior to IMI for the treatment of complicated urinary tract infections (cUTI), including acute pyelonephritis (Table 2-cefiderocol) [9•]. The subgroup analysis of patients with ESBLproducing organisms $(n=106)$ favored cefiderocol, although the types of $\beta$ lactamase were not distinguished [9•]. Phase III studies are in progress to evaluate the efficacy of cefiderocol for treatment of CRE infections (including healthcare-associated bacterial pneumonia (HABP), ventilator-associated bacterial pneumonia (VABP), cUTI, and bloodstream infections (BSI)) compared to best available therapy (BAT). It is also being studied in combination with linezolid compared to meropenem plus linezolid for the treatment of nosocomial pneumonia.

CZA is a combination of a third-generation cephalosporin with a non- $\beta$-lactam, diazabicyclooctane $\beta$-lactamase inhibitor (Table 1 ). Avibactam extends activity to ceftazidime-resistant Enterobacteriaceae via potent inhibition of class A (ESBL, $\mathrm{KPC}), \mathrm{C}(\mathrm{amp}-\mathrm{C})$, and some class $\mathrm{D} \beta$-lactamases, but it is not effective against 
Table 1. Antibiotics recently approved or in development with activity against gram-negative organisms

\begin{tabular}{|c|c|c|c|}
\hline Drug & Drug class & Clinical trials & Comments \\
\hline Cefiderocol & $\begin{array}{l}\text { Catechol siderophore } \\
\text { cephalosporin with activity } \\
\text { against all } \beta \text {-lactamase classes } \\
\text { and activity against } \\
\text { carbapenem-resistant } \\
\text { Enterobacteriaceae and } \\
\text { P. aeruginosa, } \text { A. baumannii, } \\
\text { S. maltophilia }\end{array}$ & $\begin{array}{l}\text { - Phase II: cUTI } \\
\text { - Current phase III: CRE } \\
\text { infections (HABP, VABP, } \\
\text { cUTI, BSI); nosocomial } \\
\text { pneumonia (HABP/VABP) }\end{array}$ & Not currently FDA approved \\
\hline $\begin{array}{l}\text { Ceftazidime/avibactam } \\
\text { (CZA) }\end{array}$ & $\begin{array}{l}\text { Third-generation cephalosporin in } \\
\text { combination with a } \beta \text {-lactamase } \\
\text { inhibitor with extended activity } \\
\text { including inhibition against } \\
\text { class A (ESBL, KPC) and C } \\
\text { (amp-C) and some class D } \\
\beta \text {-lactamases }\end{array}$ & $\begin{array}{l}\text { - Phase III: cIAI with } \\
\text { metronidazole } \\
\text { - Phase III: cUTI including } \\
\text { acute pyelonephritis } \\
\text { - Phase III: HABP/VABP } \\
\text { - Current phase II: febrile } \\
\text { neutropenia (terminated), } \\
\text { pediatric patients with cIAI } \\
\text { and cUTI }\end{array}$ & $\begin{array}{l}\text { FDA approved in } 2015 \text { for } \\
\text { CIAI in combination } \\
\text { with metronidazole and } \\
\text { cUTI including } \\
\text { pyelonephritis } \\
\text { Approved for HABP/VABP in } \\
2018\end{array}$ \\
\hline Ceftobiprole medocaril & $\begin{array}{l}\text { Water soluble prodrug of a } \\
\text { fifth-generation cephalosporin } \\
\text { with activity against MRSA and } \\
\text { ampicillin-sensitive } \\
\text { Enterococcus spp. }\end{array}$ & $\begin{array}{l}\text { - Phase III: ABSSSI } \\
\text { - Phase III: CABP, HABP, } \\
\text { VABP } \\
\text { - Current phase III: } \\
\text { S. aureus BSI }\end{array}$ & $\begin{array}{l}\text { Approved in several } \\
\text { countries for ABSSSI, } \\
\text { not currently FDA } \\
\text { approved }\end{array}$ \\
\hline $\begin{array}{l}\text { Ceftolozane/tazobactam } \\
\text { (C/T) }\end{array}$ & $\begin{array}{l}\text { Antipseudomonal } \beta \text {-lactam in } \\
\text { combination with a } \beta \text {-lactamase } \\
\text { inhibitor, with activity against } \\
\text { class } A \text { and } C \text {-lactamases } \\
\text { (most ESBL, amp-C) and some } \\
\text { anaerobes }\end{array}$ & $\begin{array}{l}\text { - Phase III: cUTI } \\
\text { including acute } \\
\text { pyelonephritis } \\
\text { - Phase III: cIAI (with } \\
\text { metronidazole) } \\
\text { - Current phase III: } \\
\text { pediatric cIAI, cUTI } \\
\text { - Current phase III: MDR } \\
\text { P. aeruginosa infections, } \\
\text { VABP (prior study for VABP } \\
\text { terminated) }\end{array}$ & $\begin{array}{l}\text { FDA approved in } 2015 \text { for } \\
\text { cUTI and cIAI (in } \\
\text { combination with } \\
\text { metronidazole) }\end{array}$ \\
\hline Delafloxacin & $\begin{array}{l}\text { Anionic fluoroquinolone with } \\
\text { increased activity against MRSA, } \\
\text { gram-positive isolates }\end{array}$ & $\begin{array}{l}\text { - Phase III: ABSSSI } \\
\text { - Current phase III: CABP, } \\
\text { uncomplicated urogenital } \\
\text { N. gonorrhoeae }\end{array}$ & $\begin{array}{l}\text { FDA approved for ABSSSI in } \\
2017 \\
\text { Does not prolong the QTC }\end{array}$ \\
\hline Eravacycline & $\begin{array}{l}\text { Fluorocycline, tetracycline class } \\
\text { with enhanced activity for } \\
\text { gram-positive (including } \\
\text { Enterococcus spp. but NOT } \\
\text { MRSA), gram-negative, and } \\
\text { anaerobes except Pseudomonas } \\
\text { spp. }\end{array}$ & $\begin{array}{l}\text { - Phase III: cIAI } \\
\text { - Phase III: cUTI (not yet } \\
\text { published, failed to show } \\
\text { noninferiority per press } \\
\text { release) } \\
\text { - Studies ongoing in } \\
\text { pediatric population }\end{array}$ & $\begin{array}{l}\text { FDA approved in } 2018 \text { for } \\
\text { cIAI }\end{array}$ \\
\hline $\begin{array}{l}\text { Imipenem/cilastatin + } \\
\text { relebactam }\end{array}$ & $\begin{array}{l}\text { Relebactam is a novel piperidine } \\
\text { analogue diazabicyclooctane } \\
\text { class } A / C \beta \text {-lactamase inhibitor. }\end{array}$ & $\begin{array}{l}\text { - Phase II: cIAI, cUTI } \\
\text { - Current phase III: cIAI, } \\
\text { HABP, VABP }\end{array}$ & Not currently FDA approved \\
\hline
\end{tabular}


Table 1. (Continued)

\begin{tabular}{|c|c|c|c|}
\hline Drug & Drug class & Clinical trials & Comments \\
\hline \multicolumn{4}{|c|}{$\begin{array}{l}\text { It does not inhibit class D } \\
\text { enzymes }\end{array}$} \\
\hline Lefamulin & $\begin{array}{l}\text { Semisynthetic pleuromutilin that } \\
\text { inhibits protein synthesis by } \\
\text { binding to the } 50 \text { S ribosome }\end{array}$ & $\begin{array}{l}\text { - Phase II: ABSSSI } \\
\text { - Current phase III: CABP }\end{array}$ & Not currently FDA approved \\
\hline Meropenem-vaborbactam & $\begin{array}{l}\text { Carbapenem in combination with } \\
\text { boronic acid } \beta \text {-lactamase } \\
\text { inhibitor }\end{array}$ & $\begin{array}{l}\text { - Phase III: cUTI } \\
\text { including acute } \\
\text { pyelonephritis } \\
\text { - Phase III: serious CRE } \\
\text { infections }\end{array}$ & $\begin{array}{l}\text { FDA approved in } 2018 \text { for } \\
\text { CUTI }\end{array}$ \\
\hline Plazomicin & $\begin{array}{l}\text { Aminoglycoside with enhanced } \\
\text { activity against isolates with } \\
\text { aminoglycoside-modifying } \\
\text { enzymes, ESBL, CRE including } \\
\text { KPC, and MBL }\end{array}$ & $\begin{array}{l}\text { - Phase II: cUTI including } \\
\text { acute pyelonephritis } \\
\text { - Current phase III: cUTI, } \\
\text { CRE infections }\end{array}$ & $\begin{array}{l}\text { FDA approved in } 2018 \text { for } \\
\text { cUTI }\end{array}$ \\
\hline Solithromycin & $\begin{array}{l}\text { Fourth-generation macrolide } \\
\text { (fluoro-ketolide) }\end{array}$ & $\begin{array}{l}\text { - Phase III: CABP } \\
\text { - Phase II: N. gonorrhoeae } \\
\text { infections } \\
\text { - Current phase II, III } \\
\text { studies for urogenital } \\
\text { N. gonorrhoeae infections }\end{array}$ & $\begin{array}{l}\text { Not currently FDA approved } \\
\text { No effect on QTc based on } \\
\text { phase } 1 \text { study data. } \\
\text { Metabolized by cyp3A4p450 }\end{array}$ \\
\hline Sulopenem & $\begin{array}{l}\text { Carbapenem (IV and PO) with } \\
\text { activity against gram-positive } \\
\text { and gram-negatives including } \\
\text { ESBL. Not active against } \\
\text { P. aeruginosa, Enterococcus } \\
\text { spp., CRE, or MRSA }\end{array}$ & $\begin{array}{l}\text { - Phase II: CABP } \\
\text { - Current phase III: cIAI }\end{array}$ & Not currently FDA approved \\
\hline \multicolumn{4}{|c|}{$\begin{array}{l}\text { cUTI = complicated urinary tract infection, } C R E=\text { carbapenem-resistant Enterobacteriaceae, } H A B P=\text { hospital-associated bacterial pneumonia, } \\
V A B P=\text { ventilator-associated bacterial pneumonia, } B S I=\text { bloodstream infection, } F D A=\text { Food and Drug Administration, } C Z A=\text { ceftazidime- } \\
\text { avibactam, } E S B L=\text { extended spectrum } \beta \text {-lactamase, } K P C=K \text { lebsiella pneumoniae carbapenemase, } C I A I=\text { complicated intraabdominal infection, } \\
M R S A=\text { methicillin-resistant Staphylococcus aureus, } A B S S S I=\text { acute bacterial skin and skin structure infection, } C A B P=\text { community-associated } \\
\text { bacterial pneumonia, } M D R=\text { multidrug resistant, } I V=\text { intravenous, } P O=\text { oral }\end{array}$} \\
\hline
\end{tabular}

class B $\beta$-lactamases (MBL) $[64,65]$. CZA is most efficacious for MDR Enterobacteriaceae, particularly K. pneumoniae and Escherichia coli including those producing ESBL, KPC, and OXA-48 [12•, 19-21, 65]. In vitro studies suggest that coexistence of mixed populations of organisms can lead to reemergence of mutated $b l a_{\mathrm{KPC}}$ after treatment via plasmids that may restore meropenem susceptibility $[25,66]$.

CZA is currently approved at a dose of $2 / 0.5 \mathrm{~g}$ every $8 \mathrm{~h}$ IV and is rapidly bactericidal [67]. It demonstrates similar PK across genders and ages, as well as in patients with cystic fibrosis [68-70]. It requires renal adjustment with maintenance of a 4:1 ceftazidime to avibactam ratio and administration after hemodialysis [71]. Subgroup analysis of a phase III trial by Mazuski et al. favored meropenem over CZA in patients with moderate renal impairment [29••]. More studies will be needed to determine efficacy in patients with renal 


\section{Table 2. Clinical studies by antibiotic}

\begin{tabular}{|c|c|c|c|}
\hline Article & Study type & Outcomes $^{1}$ & Comments \\
\hline \multicolumn{4}{|l|}{ Cefiderocol } \\
\hline $\begin{array}{l}\text { Portsmouth et al. } \\
\text { 2018. Lancet } \\
\text { Infectious } \\
\text { Diseases }[9 \bullet]\end{array}$ & $\begin{array}{l}\text { Phase II double-blind multicenter } \\
\text { international noninferiority RCT: } \\
\text { cefiderocol vs IMI } 1 \mathrm{~g} \text { every } 8 \mathrm{~h} \text { for cUTI } \\
\text { or pyelonephritis due to gram-negative } \\
\text { organisms }\end{array}$ & $\begin{array}{l}\text { - Noninferiority for composite cure at } \\
\text { TOC ( } 7 \text { days after EOT): } 183 / 252 \\
(72.6 \%) \text { cefiderocol vs } 65 / 119 \\
(54.6 \%) \text { IMI ( } p=0.0004 \text { ) (in } \\
\text { mMIT²) } \\
\text { - Microbiologic response at TOC } 184 / 252 \\
\text { (73\%) cefiderocol vs } 67 / 119(56.3 \%) \\
\text { IMI } \\
\text { - Clinical response at TOC } 226 / 252 \\
\text { (89.7\%) cefiderocol vs } 104 / 119(87.4 \%) \\
\text { IMI } \\
\text { - Relapse } 12 / 252 \text { ( } 4.8 \%) \text { cefiderocol vs } \\
\text { 12/119 }(10.1 \%) \text { at } 14 \text { days after EOT } \\
\text { - Composite response at TOC for ESBL } \\
44 / 70(62.9 \%) \text { cefiderocol vs } 17 / 36 \\
\text { (47.2\%) IMI }\end{array}$ & $\begin{array}{l}\text { Infection type: cUTI and acute } \\
\quad \text { pyelonephritis } \\
\text { Organisms: mostly } E \text {. coli }(223 / 371) \text {, } \\
\text { K. pneumoniae ( } 71 / 371), P \text {. aeruginosa } \\
(23 / 371), P \text {. mirabilis }(18 / 371) \text {, and } \\
\text { Enterobacter spp. (18/371) } \\
\text { Resistance mechanisms: ESBL (106/371) } \\
\text { Other comments: higher number of } \\
\text { patients with pyelonephritis in the } \\
\text { cefiderocol group } \\
\text { AE: } 41 \% \text { cefiderocol, with } 14 / 300(5 \%) \\
\text { serious events vs } 12 / 148(8 \%) \text { IMI. GI } \\
\text { most common, C. difficile colitis in } \\
1 / 300 \text { vs } 2 / 148 \text { IMI }\end{array}$ \\
\hline \multicolumn{4}{|c|}{ Ceftazidime/avibactam (CZA) } \\
\hline $\begin{array}{l}\text { De la Calle et al. } \\
\text { 2018. Int J } \\
\text { Antimicrob } \\
\text { Agents [10] }\end{array}$ & $\begin{array}{l}\text { Retrospective observational study: } \\
\text { patients with OXA-48 and } \\
\text { OXA-48-like infection received CZA } \\
\text { as compassionate use for at least } \\
72 \mathrm{~h}\end{array}$ & $\begin{array}{l}\text { - Clinical cure at } 30 \text { days } 15 / 24 \\
(62.5 \%)(2 \text { deceased, } 4 \text { with } \\
\text { persistent infection, } 3 \text { with } \\
\text { recurrence) } \\
\text { - } 30 \text {-day attributable mortality } 2 / 24 \\
(8.3 \%) \\
\text { - } 90 \text {-day attributable mortality } 3 / 24 \\
(12.5 \%) \\
\text { - No difference in } 90 \text {-day mortality for } \\
\text { monotherapy vs combination; no } \\
\text { statistical difference in age, SOFA, or } \\
\text { severe sepsis/shock on presentation }\end{array}$ & $\begin{array}{l}\text { Infection type: IAI (7/24), cUTI } \\
\quad(6 / 24), \text { HABP/NABP }(5 / 24) \text {, } \\
\text { ABSSSI/OM (4/24), and CNS }(1 / 24) \\
\text { Organisms: } K \text {. pneumoniae }(23 / 24) \text { and } \\
\text { E. coli }(1 / 24) \\
\text { Resistance mechanisms: OXA-48 (24/24) } \\
\text { or CTX-M15 (22/24). All ST101 } \\
\text { Other comments: } 15 / 23 \text { CZA used as } \\
\text { initial treatment } \\
\text { Combination therapy (9/23) } \\
\text { Other agents used: amikacin, colistin, } \\
\text { and tigecycline }\end{array}$ \\
\hline $\begin{array}{l}\text { Gofman et al. } \\
\text { 2018. Am J } \\
\text { Health Syst } \\
\text { Pharm [11] }\end{array}$ & $\begin{array}{l}\text { Case report: } 32 \text {-year-old male with } \\
\text { intracranial hemorrhage due to } \\
\text { traumatic injury with VP shunt and } \\
\text { carbapenem-resistant } \\
K . \text { pneumoniae and } P \text {. aeruginosa } \\
\text { ventriculitis }\end{array}$ & $\begin{array}{l}\text { - Treated with CZA } 2.5 \mathrm{mg} \text { IV every } \\
8 \mathrm{~h} \times 6 \text { weeks and intrathecal } \\
\text { amikacin } \times 4 \text { weeks } \\
\text { - CSF sterilized within } 3 \text { days, and } \\
\text { ventriculitis resolved }\end{array}$ & Resistance mechanisms: CRE \\
\hline $\begin{array}{l}\text { Sousa et al. } \\
\text { 2018. J } \\
\text { Antimicrob } \\
\text { Chemo }[12 \bullet]\end{array}$ & $\begin{array}{l}\text { Prospective observational study in } \\
\text { Spain: CZA for salvage therapy for } \\
\text { treatment of infections due to } \\
\text { OXA-48 carbapenemase-producing } \\
\text { Enterobacteriaceae }\end{array}$ & $\begin{array}{l}\text { - Clinical cure } 44 / 57(77.2 \%) \\
\text { - Microbiologic cure } 37 / 57(64.9 \%) \\
\text { - } 14 \text {-day all-cause mortality } 8 / 57(14 \%) \\
\text { - } 30 \text {-day all-cause mortality } 13 / 57 \\
(22.8 \%) \\
\text { - } 30 \text {-day infection-related mortality } \\
8 / 57(14 \%) \\
\text { - } 90 \text {-day recurrence } 6 / 57(10.5 \%) \\
\text { - On multivariate analysis, only } \\
\text { INCREMENT-CP score associated with } \\
\text { all-cause mortality }(p=0.001)\end{array}$ & $\begin{array}{l}\text { Infection type: BSI }(26 / 57) \text {, cIAI } \\
\quad(16 / 57), \text { HABP }(15 / 57) \text {, and cUTI } \\
\quad(14 / 57) \\
\text { Organisms: mostly } K . \text { pneumoniae } \\
\text { (54/57) } \\
\text { Resistance mechanisms: OXA-48 ( } 57 / 57) \\
\text { Other comments: combination therapy } \\
(11 / 57) \\
\text { Other agents used: colistin (IV or } \\
\text { inhaled), tigecycline, amikacin, and IMI }\end{array}$ \\
\hline $\begin{array}{l}\text { Rodriguez-Nuñez } \\
\text { et al. 2018. J } \\
\text { Glob Antimicrob } \\
\text { Resistance [13] }\end{array}$ & $\begin{array}{l}\text { Retrospective study in Spain: CZA for } \\
\text { compassionate use in MDR or XDR } \\
\text { Pseudomonas aeruginosa infections }\end{array}$ & $\begin{array}{l}\text { - Clinical cure } 4 / 8(50 \%) \\
\text { - Microbiologic cure } 4 / 8(50 \%) \\
\text { - } 30 \text {-day all-cause mortality } 1 / 8(12.5 \%) \\
\text { - } 90 \text {-day all-cause mortality } 3 / 8(37.5 \%)\end{array}$ & $\begin{array}{l}\text { Infection type: } \\
\text { HABP/VABP } \\
(4 / 8) \text {, tracheobronchitis }(1 / 8), 0 M \\
(1 / 8), \text { CNS }(1 / 8) \text {, and BSI (1/8) } \\
\text { Organisms: } \text { P. aeruginosa }(8 / 8) \\
\text { Resistance mechanisms: OXA-48 (1/8), } \\
\text { ESBL (1/8), or XDR (3/8) } \\
\text { Other comments: combination therapy } \\
\text { (5/8) } \\
\text { Other agents used: inhaled amikacin, } \\
\text { inhaled colistin, tobramycin, and } \\
\text { ciprofloxacin }\end{array}$ \\
\hline $\begin{array}{l}\text { Mittal et al. } \\
\text { 2018. BMJ Case } \\
\text { Reports [14] }\end{array}$ & $\begin{array}{l}\text { Case report: } 42 \text {-year-old male with } \\
\text { orthopedic infection due to injury } \\
\text { that occurred in Bangladesh with } \\
\text { OXA-181 and NDM-producing } \\
\text { K. pneumoniae }\end{array}$ & $\begin{array}{l}\text { - Achieved clinical and microbiologic } \\
\text { cure with aztreonam/CZA } \\
\text { combination and surgical } \\
\text { debridement }\end{array}$ & $\begin{array}{l}\text { Infection type: OM } \\
\text { Resistance mechanisms: OXA-181, } \\
\text { NDM-1, TEM-1b, SHV-28, 0XA-9, and } \\
\text { CTX-M-15 } \\
\text { Other comments: coinfected with } \\
\text { Rhizopus spp. }\end{array}$ \\
\hline
\end{tabular}




\begin{tabular}{|c|c|c|c|}
\hline \multirow[t]{2}{*}{ Article } & Study type & Outcomes $^{1}$ & Comments \\
\hline & & & $\begin{array}{l}\text { Found synergy with aztreonam and CZA } \\
\text { when tested MIC alone and in } \\
\text { combination with fractional inhibitory } \\
\text { concentration index } \leq 0.5 \text { (MIC of } \\
\text { aztreonam reduced from } 64 \text { to } 4 \mu \mathrm{g} / \mathrm{mL} \text { ) }\end{array}$ \\
\hline $\begin{array}{l}\text { Santeveechi } \\
\text { et al. 2018. Int } \\
\text { J Antimicrob } \\
\text { Agents [15] }\end{array}$ & $\begin{array}{l}\text { Retrospective case series: treating } \\
\text { resistant gram-negative organisms } \\
\text { other than } K . \text { pneumoniae }\end{array}$ & $\begin{array}{l}\text { - Microbiologic cure } 6 / 9(66.7 \%) \\
\text { - Clinical success } 7 / 10(70 \%) \\
\text { - } 30 \% 30 \text { days in hospital mortality }\end{array}$ & $\begin{array}{l}\text { Infection type: LRTI (6/13), ABSSSI } \\
\quad(3 / 13), \text { BSI }(2 / 13) \text {, and cIAI ( } 2 / 13) \\
\text { Organisms: mostly P. aeruginosa ( } 8 / 13) \\
\text { and Citrobacter spp. (4/13) } \\
\text { Polymicrobial }(6 / 13) \\
\text { Resistance mechanisms: all MDR } \\
\text { Other comments: combination therapy } \\
\text { (5/10) } \\
\text { Other agents used: tigecycline, } \\
\text { aminoglycosides, colistin, ciprofloxacin, } \\
\text { TMP/SMX, and carbapenems }\end{array}$ \\
\hline $\begin{array}{l}\text { Torres et al. } \\
\text { 2018. (REPROVE } \\
\text { trial) Lancet } \\
\text { Infectious } \\
\text { Diseases [16••] }\end{array}$ & $\begin{array}{l}\text { Multicenter, international } \\
\text { double-blind phase III } \\
\text { noninferiority RCT: CZA vs } \\
\text { meropenem for HABP/VABP }\end{array}$ & $\begin{array}{l}\text { - Noninferior for clinical cure at TOC } \\
(28-32 \text { days post randomization) } \\
245 / 356(68.8 \%) \text { CZA vs } 270 / 370 \\
(73 \%) \text { meropenem } \\
\text { - No difference in } 28 \text {-day all-cause } \\
\text { mortality } 38 / 405(9 \%) \text { CZA vs } 30 / 403 \\
\text { (7\%) meropenem } \\
\text { - Microbiologic cure at TOC } 95 / 171 \\
(55.6 \%) \text { vs } 118 / 184(64.1 \%) \text { in } \text { mMITT }^{3}\end{array}$ & $\begin{array}{l}\text { Infection type: HABP/VABP } \\
\text { Organisms: (mMITT) mostly } \\
\text { K. pneumoniae }(130 / 355), P \text {. aeruginosa } \\
(105 / 355), \text { E. cloacae }(48 / 355) \text {, } \\
\text { H. influenzae (41/355), E. coli } \\
\text { (37/355), S. marcescens }(28 / 355) \text {, } \\
\text { P. mirabilis (26/355), and E. aerogenes } \\
\text { (16/355) } \\
\text { Polymicrobial }(152 / 355)\end{array}$ \\
\hline $\begin{array}{l}\text { Van Duin et al. } \\
\text { 2018. CID [17•] }\end{array}$ & $\begin{array}{l}\text { Multicenter prospective observational } \\
\text { study: colistin vs CZA for CRE } \\
\text { infections }\end{array}$ & $\begin{array}{l}\text { - All-cause } 30 \text {-day mortality } 3 / 38 \text { (8\%) } \\
\text { CZA vs } 33 / 99(33 \%) \text { colistin } \\
\text { - All-cause } 30 \text {-day mortality following } \\
\text { adjustment } 9 \text { vs } 52 \% \text { ( } p=0.001) \\
\text { - Overall better outcome with CZA by } \\
64 \%\end{array}$ & $\begin{array}{l}\text { Infection type: BSI (63/137), RTI } \\
\quad(33 / 137), \text { UTI (19/137), and wound } \\
\quad(14 / 137) \\
\text { Organisms: } K \text {. pneumoniae (133/137) } \\
\text { and Enterobacter spp. }(4 / 137) \\
\text { Resistance mechanisms }{ }^{4}: 52 / 137 \mathrm{KPC} \text {. } \\
\text { No OXA-48, VIM, NDM, or IMP } \\
\text { Other comments: combination therapy } \\
\text { 24/38 CZA and 93/99 colistin } \\
\text { Other agents used: tigecycline, } \\
\text { aminoglycosides, carbapenems, } \\
\text { TMP/SMX, and fosfomycin }\end{array}$ \\
\hline $\begin{array}{l}\text { Mariano et al. } \\
\text { 2017. Case Rep } \\
\text { Infect Disease } \\
\text { [18] }\end{array}$ & $\begin{array}{l}\text { Case report: } 66 \text {-year-old male with KPC } \\
\text { Klebsiella spp. epidural abscess with } \\
\text { hardware present and BSI }\end{array}$ & $\begin{array}{l}\text { - Clinical and microbiological cure with } \\
\text { CZA monotherapy }\end{array}$ & $\begin{array}{l}\text { Previously had failed meropenem and } \\
\text { tigecycline }\end{array}$ \\
\hline $\begin{array}{l}\text { King et al. } 2017 . \\
\text { Antimicrob } \\
\text { Agents } \\
\text { Chemother [19] }\end{array}$ & $\begin{array}{l}\text { Multicenter retrospective } \\
\text { observational study: CZA for } \\
\text { carbapenem-resistant } \\
\text { Enterobacteriaceae infections }\end{array}$ & $\begin{array}{l}\text { - In-hospital mortality } 19 / 60(32 \%) \\
\text { - Clinical cure } 18 / 35(51 \%) \text { with CZA } \\
\text { known sensitivity and } 13 / 24(54 \%) \text { with } \\
\text { unknown sensitivity } \\
\text { - No mortality difference with } \\
\text { monotherapy vs combination }\end{array}$ & $\begin{array}{l}\text { Infection type: BSI }(23 / 60) \text {, LRTI } \\
(16 / 60), \text { UTI }(17 / 60) \text {, wound } \\
(8 / 60), \text { IAI }(4 / 60) \text {, and 0M/joint } \\
(2 / 60) \\
\text { Organisms: mostly K. pneumoniae } \\
(50 / 60) \\
\text { Other comments: included } 25 \% \text { SOT } \\
\text { Combination therapy }(27 / 60) \\
\text { Other agents used: aminoglycosides, } \\
\text { polymyxin, and tigecycline }\end{array}$ \\
\hline $\begin{array}{l}\text { Castòn et al. } \\
\text { 2017. Int J } \\
\text { Infect Dis [20] }\end{array}$ & $\begin{array}{l}\text { Retrospective multicenter } \\
\text { observational: outcomes in patients } \\
\text { with hematologic malignancy and } \\
\text { BSI due to carbapenemase } \\
\text { producing organism }\end{array}$ & $\begin{array}{l}\text { - CZA with higher clinical cure } 6 / 8 \\
(75 \%) \text { vs } 8 / 23(35 \%)(p=0.23) \\
\text { alternative } \\
\text { - No difference in } 30 \text {-day mortality } 2 / 8 \\
(25 \%) \text { CZA vs } 12 / 23(52 \%) \text { BAT }(p= \\
0.24)\end{array}$ & $\begin{array}{l}\text { Infection type: BSI (30/31) } \\
\text { Source: primary BSI }(16 / 31), \text { RTI (8/31), } \\
\text { CLABSI ( } 7 / 31) \text {, wound/ulcer }(2 / 31) \text {, and } \\
\text { UTI }(1 / 31) \\
\text { Organism type: mostly } K . \text { pneumoniae } \\
\text { ( } 25 / 31) \\
\text { Resistance mechanisms: 0XA-48 (19/31) } \\
\text { or KPC (12/31) } \\
\text { Other comments: combination therapy } \\
\text { ( } 25 / 31) \\
\text { Other agents used: aminoglycoside, } \\
\text { carbapenems, fosfomycin, tigecycline, } \\
\text { and colistin }\end{array}$ \\
\hline
\end{tabular}




\begin{tabular}{|c|c|c|c|}
\hline Article & Study type & Outcomes $^{1}$ & Comments \\
\hline $\begin{array}{l}\text { Krapp et al. } \\
\text { 2017. Int J } \\
\text { Antimicrob } \\
\text { Agents [21] }\end{array}$ & $\begin{array}{l}\text { Retrospective review: CRE } \\
\text { K. pneumoniae infections treated } \\
\text { with CZA }\end{array}$ & $\begin{array}{l}\text { - Clinical cure } 3 / 6(50 \%) \\
\text { - Mortality } 3 / 6(50 \%)\end{array}$ & $\begin{array}{l}\text { Infection type: RTI }(2 / 6), \text { IAI }(2 / 6) \text {, } \\
\text { CUTI }(1 / 6) \text {, and wound }(1 / 6) \\
\text { Organisms: } \text {. pneumoniae }(6 / 6), \text { E. coli } \\
(2 / 6), \text { VRE }(1 / 6), \text { MRSA }(1 / 6) \text {, and } \\
\text { M. morganii }(1 / 6) \\
\text { Resistance mechanisms: all with OXA-9, } \\
\text { KPC, TEM, and SHV } \\
\text { Other comments: combination therapy } \\
(4 / 6) \\
\text { Other agents used: inhaled polymyxin B } \\
\text { and tigecycline }\end{array}$ \\
\hline $\begin{array}{l}\text { Qin et al. } 2017 . \\
\text { Int J Antimicrob } \\
\text { Agents [22••] }\end{array}$ & $\begin{array}{l}\text { Phase III international, multicenter, } \\
\text { double-blind noninferiority RCT } \\
\text { (RECLAIM 3): CZA + metronidazole } \\
\text { vs meropenem for cIAI in Asia }\end{array}$ & $\begin{array}{l}\text { - Clinical cure noninferior at TOC } \\
(28-35 \text { days post-randomization) } \\
\text { in CE population } 166 / 177(93.8 \%) \\
\text { CZA vs 173/184 }(94 \%) \text { meropenem } \\
(p<0.001) \\
\text { - Similar results for ceftazidime-NS } \\
\text { isolates }\end{array}$ & $\begin{array}{l}\text { Infection type: cIAI } \\
\text { Organisms: }\left(\text { mITT }{ }^{3}\right) \text { mostly } E . \text { coli } \\
(173 / 295), K . \text { pneumoniae }(63 / 295), \\
\text { and } P \text {. aeruginosa }(37 / 295)\end{array}$ \\
\hline $\begin{array}{l}\text { Shields et al. } \\
2017 . \\
\text { Antimicrob } \\
\text { Agents } \\
\text { Chemother [23] }\end{array}$ & $\begin{array}{l}\text { Retrospective observational study: } \\
\text { patients with carbapenem-resistant } \\
\text { K. pneumoniae bacteremia }\end{array}$ & $\begin{array}{l}\text { - Higher clinical success with CZA } \\
11 / 13(86 \%) \text { than carbapenem plus } \\
\text { aminoglycoside } 12 / 25(48 \%)(p= \\
0.04) \text {, or carbapenem plus colistin } \\
12 / 30(40 \%)(p=0.009) \text { or other } \\
15 / 41(36.6 \%)(p=0.006) \\
\text { - } 90 \text {-day all-cause mortality } 1 / 13(7.7 \%) \\
\text { CZA vs } 11 / 25(24.4 \%) \text { vs } 11 / 30(36.7 \%) \\
\text { vs } 21 / 41(51.2 \%) \\
\text { - Primary BSI (OR } 4.5, p=0.01) \text { and } \\
\text { receipt of CZA (OR } 8.64, p=0.01) \text { were } \\
\text { independent predictors of clinical success }\end{array}$ & $\begin{array}{l}\text { Infection type: BSI (109/109) } \\
\text { Source: primary (28/109), IAI (50/109), } \\
\text { RTI (14/109), UTI (14/109), and ABSSSI } \\
(3 / 109) \\
\text { Organisms: } \text { K. pneumoniae (109/109) } \\
\text { Resistance mechanisms: mostly KPC } \\
\text { (106/109) } \\
\text { Other comments: combination therapy } \\
\text { (5/13) } \\
\text { Other agents used: gentamycin }\end{array}$ \\
\hline $\begin{array}{l}\text { Mendes et al. } \\
2017 . \\
\text { Antimicrob } \\
\text { Agents } \\
\text { Chemother } \\
{[24 \bullet \text { ] }}\end{array}$ & $\begin{array}{l}\text { International multicenter phase III } \\
\text { RCT: characterized } \beta \text {-lactamase } \\
\text { content of baseline pathogens } \\
\text { isolated from patients with cIAI } \\
\text { enrolled in two phase III RCTs } \\
\text { comparing CZA to meropenem }\end{array}$ & $\begin{array}{l}\text { - Similar clinical cure rates at TOC } \\
(28-35 \text { days post-randomization) } \\
\text { in } \text { mMITT }^{3} \text { for CZA } 337 / 413(81.6 \%) \\
\text { and meropenem } 349 / 410(85.1 \%) \\
\text { - Similar cure rates seen with MIC screen } \\
\text { positive pathogens } 49 / 56(87.5 \%) \text { vs } \\
64 / 74(86.5 \%) \text { and negative } 272 / 331 \\
(82.2 \%) \text { vs } 275 / 320(85.9 \%) \text { for } \\
\beta \text {-lactamases } \\
\text { - Cure for ESBL/carbapenemase } 38 / 42 \\
\text { (90.5\%) vs } 47 / 55(85.4 \%) \\
\text { - Cure for amp-C } 9 / 12(75 \%) \text { vs } 13 / 15 \\
(86.7 \%) \\
\text { - All } P \text {. aeruginosa isolates with positive } \\
\text { MIC screen }(6 / 6) \text { had clinical cure }\end{array}$ & $\begin{array}{l}\text { Infection type: cIAI } \\
\text { Organisms: mostly E. coli (81/138), } \\
\text { K. pneumoniae (28/138), Enterobacter } \\
\text { spp. (12/138), P. aeruginosa (6/138), } \\
\text { P. mirabilis }(5 / 138), \text { C. freundii }(3 / 138), \\
\text { and S. marcescens (3/138) } \\
\text { Resistance mechanisms: ESBL }(99 / 138) \text {, } \\
\text { OXA (61/138), Amp-C (16/138), CMY } \\
(14 / 138), \text { SHV (5/138), PER-1 (4/138), } \\
\text { NDM (3/138), or TEM (2/138) } \\
\text { CTX-M variants alone }(41 / 138) \text { or in } \\
\text { combination with OXA-1/30 (49/138) } \\
\text { were most common } \beta \text {-lactamases in } \\
\text { Enterobacteriaceae }\end{array}$ \\
\hline $\begin{array}{l}\text { Shields et al. } \\
2017 . \\
\text { Antimicrob } \\
\text { Agents } \\
\text { Chemother [25] }\end{array}$ & $\begin{array}{l}\text { Case series: } 3 \text { case reports of patients } \\
\text { developing CZA resistance in } \\
\text { K. pneumoniae isolates on CZA } \\
\text { therapy }\end{array}$ & $\begin{array}{l}\text { - CZA resistance due to plasmid bla } \text { KIPC-3 }_{\text {a }} \\
\text { within } 10-19 \text { days of treatment } \\
\text { - All } 3 \text { patients had disease recurrence }\end{array}$ & $\begin{array}{l}\text { Infection type: cUTI (2/3), LRTI (2/3), } \\
\quad \text { and cIAI }(1 / 3) \\
\text { Organisms: } K \text {. pneumoniae (3/3) } \\
\text { Resistance mechanisms: all TEM-1, } \\
\text { SHV-11, OXA-9, and KPC-3. No NDM, } \\
\text { MBL, and OXA-48 }\end{array}$ \\
\hline $\begin{array}{l}\text { Shields et al. } \\
\text { 2016. CID [26] }\end{array}$ & $\begin{array}{l}\text { Retrospective observational study: } \\
\text { patients with CRE treated with CZA }\end{array}$ & $\begin{array}{l}\text { - 30-day survival } 28 / 37(76 \%) \\
\text { - } 90 \text {-day survival } 23 / 37(62 \%) \\
\text { - Clinical success } 22 / 37(59 \%) \\
\text { - No difference in monotherapy } 15 / 26 \\
(58 \%) \text { vs combination therapy } 7 / 11 \\
(64 \%) \\
\text { - Lower success with patients requiring } \\
\text { continuous renal replacement therapy } \\
(p=0.03) \\
\text { - SOFA scores lower in patients with } \\
\text { clinical success ( } 5.2 \text { vs } 8.8, p=0.047) \\
\text { - Microbiologic failure in } 10 / 37(20 \%)\end{array}$ & $\begin{array}{l}\text { Infection type: HABP/NABP }(12 / 37) \text {, } \\
\text { primary BSI (10/37), IAI (4/37), } \\
\text { ABSSSI (4/37), pyelonephritis (4/37), } \\
\text { mediastinitis (1/37), empyema } \\
\text { (1/37), and ventriculitis (1/37) } \\
\text { Organisms: K. pneumoniae (31/37), } \\
\text { E. coli (3/37), E. cloacae }(2 / 37), \text { and } \\
\text { E. aerogenes (1/37) } \\
\text { Resistance mechanisms: KPC (29/37). No } \\
\text { NDM, VIM, or OXA } \\
\text { Other comments: combination therapy } \\
\text { (11/37) } \\
\text { Other agents used: gentamycin } \\
\text { (IV/inhaled), colistin (IV/intrathecal), } \\
\text { and tigecycline } \\
\text { 11 SOT included }\end{array}$ \\
\hline
\end{tabular}




\begin{tabular}{|c|c|c|c|}
\hline Article & Study type & Outcomes $^{1}$ & Comments \\
\hline $\begin{array}{l}\text { Carmeli et al. } 2016 . \\
\text { Lancet ID. } \\
\quad \text { (REPRISE) }[27 \bullet]\end{array}$ & $\begin{array}{l}\text { Randomized open-label phase III RCT: CZA } \\
\text { versus BAT for the treatment of cIAI } \\
\text { and CUTI due to ceftazidime-resistant } \\
\text { Enterobacteriaceae or } P \text {. aeruginosa }\end{array}$ & $\begin{array}{l}\text { - Clinical cure at TOC (mMITT) 140/154 } \\
(91 \%) \text { CZA vs BAT 135/148 (91\%) } \\
\text { overall } \\
\text { - Clinical cure at TOC for cUTI 132/144 } \\
(92 \%) \text { CZA vs } 129 / 137(94 \%) \text { BAT } \\
\text { - Clinical cure at TOC for cIAI 8/10 (90\%) } \\
\text { CZA + metronidazole vs } 6 / 11(55 \%) \text { BAT } \\
\text { - Clinical cure at TOC for cIAI 8/10(90\%) } \\
\text { CZA + metronidazole vs } 6 / 11(55 \%) \text { BAT }\end{array}$ & $\begin{array}{l}\text { Infection type: cUTI (281/302), cIAI } \\
\quad(21 / 302) \\
\text { Mostly E. coli 126/302, K. pneumoniae } \\
(128 / 302), P \text {. aeruginosa }(21 / 302) \text { and } \\
\text { E. cloacae }(18 / 302) \\
97 \% \text { BAT group received a carbapenem } \\
\text { antibiotic ( } 96 \% \text { monotherapy) } \\
\text { Median duration of treatment was } 10 \text { days } \\
\text { in cUTI and } 10-12 \text { days in cIAI groups }\end{array}$ \\
\hline $\begin{array}{l}\text { Wagenlehner } \\
\text { et al. 2016. CID } \\
{[28 \bullet \bullet}\end{array}$ & $\begin{array}{l}\text { Phase III international double-blind } \\
\text { noninferiority RCT (RECAPTURE): } \\
\text { CZA vs doripenem for treatment of } \\
\text { hospitalized patients with cUTI and } \\
\text { pyelonephritis requiring IV therapy }\end{array}$ & $\begin{array}{l}\text { - Noninferior for symptomatic } \\
\text { resolution at day } 5 \text { in mMITT }{ }^{2,3} \\
276 / 393(70.2 \%) \text { CZA vs } 276 / 417 \\
(66.2 \%) \text { doripenem } \\
\text { - Investigator determined cure at TOC } \\
\text { (21-25 days post-randomization) } \\
355 / 393(90.3 \%) \text { vs } 377 / 417(90.4 \%) \\
\text { doripenem, LFU ( } 45-52 \text { days) } 335 / 393 \\
\text { (85.2\%) vs } 350 / 417 \text { ( } 83.9 \%) \\
\text { - Noninferior and superior for } \\
\text { per-patient favorable microbiologic } \\
\text { eradication at TOC } 304 / 393 \text { ( } 77.4 \%) \text { vs } \\
296 / 417 \text { (71\%) } \\
\text { - Combined patient assessed symptom } \\
\text { resolution and favorable microbiologic } \\
\text { response at T0C } 280 / 393 \text { ( } 71.2 \%) \text { vs } \\
269 / 417(64.5 \%)\end{array}$ & $\begin{array}{l}\text { Infection type: cUTI and acute } \\
\quad \text { pyelonephritis } \\
\text { Organisms: mostly E. coli }(598 / 810) \text {, } \\
\text { K. pneumoniae }(100 / 810), P \text {. aeruginosa } \\
\text { (38/810), P. mirabilis }(30 / 810) \text {, and } \\
\text { E. cloacae (24/810) } \\
\text { Resistance mechanisms: ESBL }(155 / 810) \\
\text { Other comments: } 57.7 \% \text { mMITT switched } \\
\text { to oral antibiotics at day } 5\end{array}$ \\
\hline $\begin{array}{l}\text { Mazuski et al. } \\
\text { 2016. CID } \\
{[29 \bullet \bullet]}\end{array}$ & $\begin{array}{l}\text { Double-blind, multicenter } \\
\text { international phase III trial } \\
\text { (RECLAIM } 1 \text { and 2): CZA + } \\
\text { metronidazole vs meropenem in } \\
\text { cIAI }\end{array}$ & $\begin{array}{l}\text { - Noninferior for cure at TOC } \\
\text { (28-35 days post-randomization), } \\
429 / 520(82.5 \%) \text { CZA vs } 444 / 523 \\
(84.9 \%), 337 / 413(81.6 \%) \text { vs } \\
349 / 410(85.1 \%) \text { mMITT } \\
\text { - Noninferior for cure at EOT } 429 / 520 \\
(82.5 \%) \text { vs } 436 / 523(83.4 \%) \text { and late } \\
\text { follow-up ( } 42-49 \text { days } \\
\text { post-randomization) } 459 / 520 \text { (88.5\%) } \\
\text { vs } 482 / 523(92.2 \%) \\
\text { - Meropenem favored in subgroup } \\
\text { analysis for moderate renal impairment } \\
\text { - For ceftazidime-resistant organisms: } \\
\text { clinical cure } 39 / 47 \text { (83\%) vs 55/64 } \\
\text { (85.9\%) }\end{array}$ & $\begin{array}{l}\text { Infection type: cIAI. BSI (36/1043) } \\
\text { Organisms: (mMITT) mostly E. coli } \\
\text { (519/823), K. pneumoniae (100/823), } \\
\text { P. aeruginosa (71/823), K. oxytoca } \\
\text { (33/823), C. freundii complex (30/823), } \\
\text { E. cloacae (32/823), P. mirabilis } \\
\text { (17/823), E. aerogenes (10/823), } \\
\text { anaerobes (140/823), and gram-positive } \\
\text { aerobes (157/823) } \\
\text { Resistance mechanisms: MBL } \\
\text { approximately } 3 \% \text {, or ESBL }(118 / 823)\end{array}$ \\
\hline $\begin{array}{l}\text { Mendes et al. } \\
2015 . \\
\text { Antimicrob } \\
\text { Agents } \\
\text { Chemother [30] }\end{array}$ & $\begin{array}{l}\text { Assessed microbiologic response in } \\
\text { ESBL vs non-ESBL from phase II RCT } \\
\text { for CZA in treatment of cUTI/acute } \\
\text { pyelonephritis and cIAI }\end{array}$ & 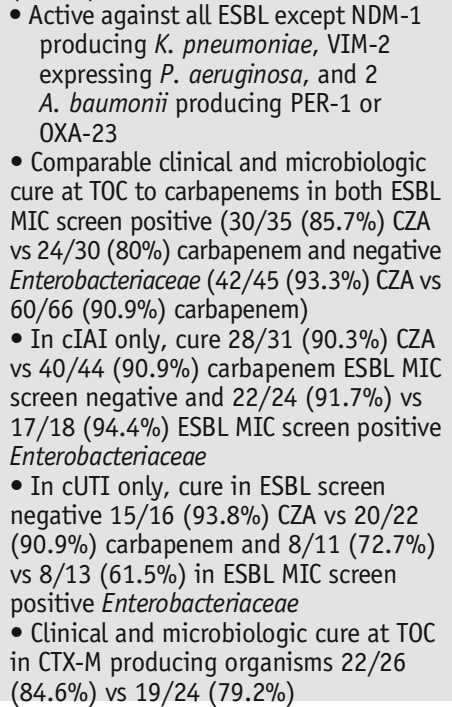 & $\begin{array}{l}\text { Infection type: cIAI (130/192) and } \\
\quad \text { cUTI (62/192) } \\
\text { Organisms (ME): mostly E. coli } \\
\text { (162/192), K. pneumoniae (16/192), } \\
\text { P. aeruginosa (9/192), and } \\
\text { Acinetobacter spp. (4/192) } \\
\text { Resistance mechanisms: ESBL 70/192, } \\
\text { CTX-M (55/192), OXA (29/192), SHV } \\
\text { (7/192), CMY (4/192), ACC (2/192), } \\
\text { NDM (1/192), VIM (1/192), PER-1 } \\
\text { (1/192), or amp-C (1/192) }\end{array}$ \\
\hline
\end{tabular}




\begin{tabular}{|c|c|c|c|}
\hline Article & Study type & Outcomes $^{1}$ & Comments \\
\hline $\begin{array}{l}\text { Lucasti et al. } \\
2013 . \\
\text { Antimicrob } \\
\text { Agents Chemo } \\
\text { [8] }\end{array}$ & $\begin{array}{l}\text { Phase II double-blind RCT: CZA + } \\
\text { metronidazole vs meropenem for } \\
\text { CIAI }\end{array}$ & $\begin{array}{l}\text { - Similar clinical response in ME } \\
62 / 68(91.2 \%) \text { vs } 71 / 76(93.4 \%) \text { at } \\
\text { TOC }(7-14 \text { days after last dose) } \\
\text { - Clinical response at TOC in mMITT } \\
70 / 85(82.4 \%) \text { vs } 79 / 89(88.8 \%) \\
\text { - Microbiologic response for ceftazidime } \\
\text { nonsusceptible } 25 / 26(96.2 \%) \text { vs } 16 / 17 \\
\text { (94.1\%) } \\
\text { - No relationship between APACHE II, } \\
\text { infection site, monomicrobial vs } \\
\text { polymicrobial }\end{array}$ & $\begin{array}{l}\text { Infection types: cIAI } \\
\text { Organisms: (ME) mostly E. coli } \\
\text { (105/144), K. pneumoniae (17/144), } \\
\text { P. aeruginosa (10/144), gram-positive } \\
\text { (22/144), and anaerobes (36/144) } \\
\text { Polymicrobial (52/144) }\end{array}$ \\
\hline \multicolumn{4}{|c|}{ Ceftobiprole medocaril } \\
\hline $\begin{array}{l}\text { Awad et al. } 2014 . \\
\text { CID [31] }\end{array}$ & $\begin{array}{l}\text { Phase III double-blind international } \\
\text { multicenter noninferiority RCT: } \\
\text { ceftobiprole vs ceftazidime + } \\
\text { linezolid for HABP/VABP }\end{array}$ & $\begin{array}{l}\text { - Noninferior for clinical cure at TOC } \\
(7-14 \text { days post-EOT) } 195 / 391 \\
(49.9 \%) \text { ceftobiprole vs } 206 / 390 \\
\quad(52.8 \%) \text { ceftazidime/linezolid } \\
\text { - Noninferior for clinical cure at TOC in } \\
\text { HABP subgroup } 171 / 287(59.6 \%) \text { vs } \\
167 / 284(58.8 \%) \\
\text { - Early improvement at day } 4 \text { with HABP } \\
221 / 287(77 \%) \text { vs } 214 / 284(75.4 \%) \\
\text { especially MRSA } 22 / 28(78.6 \%) \text { vs } 19 / 32 \\
\text { ( } 59.4 \%) \\
\text { - Could not demonstrate noninferiority } \\
\text { for VABP } 24 / 104(23.1 \%) \text { vs } 39 / 106 \\
\text { ( } 36.8 \%) \\
\text { - Microbiological eradication at TOC } \\
\text { (mMITT) } 105 / 269(39 \%) \text { vs } 127 / 267 \\
\text { (47.6\%) }\end{array}$ & $\begin{array}{l}\text { Infection type: HABP (571/781), VABP } \\
\text { (210/781), and BSI (86/781) } \\
\text { Organisms (ME): mostly S. aureus } \\
\text { (141/332) (MRSA 86/332), } \\
\text { P. aeruginosa }(61 / 332), \text { K. pneumoniae } \\
\text { (39/332), E. coli (34/332), A. baumanii } \\
\text { (31/332), S. pneumoniae (26/332), } \\
\text { Enterobacter spp. (21/332), } \\
\text { H. influenzae (18/332), Proteus spp. } \\
\text { (17/332), and Serratia spp. (15/332) } \\
\text { Polymicrobial } 187 / 332 \\
\text { Other comments: overall high severity of } \\
\text { illness, 41\% with APACHE II > 15 in each } \\
\text { group } \\
\text { AE: hyponatremia (4.4\%), diarrhea } \\
\text { (3.1\%), and dysgeusia (1.3\%) }\end{array}$ \\
\hline $\begin{array}{l}\text { Nicholson et al. } \\
\text { 2012. Int J } \\
\text { Antimicrob } \\
\text { Agents [32] }\end{array}$ & $\begin{array}{l}\text { Double-blind international, } \\
\text { multicenter phase III noninferiority } \\
\text { RCT: ceftobiprole vs ceftriaxone + } \\
\text { linezolid for CABP (not atypical) }\end{array}$ & $\begin{array}{l}\text { - Noninferior for clinical cure } 240 / 314 \\
(76.4 \%) \text { ceftobiprole vs } 257 / 324 \\
(79.3 \%) \text { ceftriaxone/linezolid } \\
\text { - Microbiologic eradication (mMITT) } \\
70 / 87(80.5 \%) \text { vs } 79 / 97(81.4 \%) \\
\text { - No significant difference in subgroup } \\
\text { analysis by clinical severity and age } \\
\text { - When switched to oral therapy, } \\
\text { eradication better in those receiving } \\
\text { cefuroxime } 41 / 41(100 \%) \text { than } \\
\text { ceftobiprole } 33 / 37(89 \%)\end{array}$ & $\begin{array}{l}\text { Infection type: CABP }(638 / 638) \text { and } \\
\text { BSI (28/638) } \\
\text { Organisms (ME): mostly S. pneumoniae } \\
\text { (64), Haemophilus spp. (38), and } \\
\text { K. pneumoniae (12) }\end{array}$ \\
\hline $\begin{array}{l}\text { Noel et al. } 2008 . \\
\text { CID [33] }\end{array}$ & $\begin{array}{l}\text { Double-blind multicenter phase II RCT: } \\
\text { ceftobiprole medocaril vs } \\
\text { vancomycin + ceftazidime for } \\
\text { complicated ABSSSI }\end{array}$ & $\begin{array}{l}\text { - Clinical cure at TOC } 448 / 547(81.9 \%) \\
\text { ceftobiprole vs } 227 / 281(80.8 \%) \\
\text { vancomycin/ceftazidime } \\
\text { - Microbiologic eradication (ME) } \\
344 / 391(88 \%) \text { vs } 177 / 199(89 \%) \\
\text { - Similar cure rates for gram-positive } \\
109 / 124(87.9 \%) \text {, gram-negative } 292 / 318 \\
(91.8 \%) \text { and across infection types }\end{array}$ & $\begin{array}{l}\text { Infection type: ABSSSI } \\
\text { Organisms: mostly MSSA (250/590), } \\
\text { MRSA (123/590), E. coli }(63 / 590) \text {, } \\
\text { P. aeruginosa (39/590), S. pyogenes } \\
\text { (32/590), E. cloacae }(22 / 590) \text {, } \\
\text { S. agalactiae (20/590), S. epidermidis } \\
\text { (15/590), K. pneumoniae (14/590), and } \\
\text { P. mirabilis (22/590) }\end{array}$ \\
\hline \multicolumn{4}{|c|}{ Ceftolozane/tazobactam (C/T) } \\
\hline $\begin{array}{l}\text { Frattari et al. } \\
\text { 2018. ID Cases } \\
\text { [34] }\end{array}$ & $\begin{array}{l}\text { Case report in Italy: } 27 \text {-year-old male } \\
\text { treated with } C / T+\text { fosfomycin for } \\
\text { rescue treatment of XDR } \\
P \text {. aeruginosa otogenous meningitis } \\
\text { with HABP, BSI }\end{array}$ & $\begin{array}{l}\text { - Initially treated with meropenem and } \\
\text { colistin, developed meningitis on } \\
\text { treatment } \\
\text { - Meningeal symptoms resolved within } 24 \mathrm{~h} \\
\text { of C/T initiation and clinically recovered } \\
\text { - Repeat CSF sterile }\end{array}$ & $\begin{array}{l}\text { Infection type: } \mathrm{HABP}, \mathrm{CNS} \text {, and BSI } \\
\text { Organisms: } P \text {. aeruginosa } \\
\text { Other comments: susceptible only to } \\
\text { colistin and C/T } \\
\text { Combination therapy with rifampin and } \\
\text { steroids }\end{array}$ \\
\hline $\begin{array}{l}\text { Bassetti et al. } \\
\text { 2018. Int J } \\
\text { Antimicrob } \\
\text { Agents [35] }\end{array}$ & $\begin{array}{l}\text { Multicenter retrospective } \\
\text { observational study: } 22 \text { hospitals in } \\
\text { Italy with C/T used to treat } \\
\text { P. aeruginosa infections }\end{array}$ & $\begin{array}{l}\text { - Clinical success } 84 / 101(83.2 \%) \\
\text { - No difference in clinical success with } \\
\text { monotherapy } 54 / 65(83.1 \%) \text { vs } \\
\text { combination } 30 / 36(83.3 \%) \text { or primary } \\
31 / 39(79.5 \%) \text { vs secondary } 53 / 62 \\
(85.5 \%) \text { treatment }(p>0.05) \\
\text { - No difference in clinical success in XDR } \\
43 / 53(81.1 \%) \text { vs non-MDR } 27 / 30(90 \%) \\
\text { or MDR } 14 / 18(77.8 \%)(p>0.05)\end{array}$ & $\begin{array}{l}\text { Infection type: HABP/VABP }(32 / 101) \text {, } \\
\text { ABSSSI }(21 / 101), \text { cUTI }(14 / 101), \\
\text { cIAI }(13 / 101), \text { OM }(9 / 101) \text {, and BSI } \\
\text { (22/101) (6 primary BSI) } \\
\text { Organisms: } P \text {. aeruginosa }(101 / 101) \\
\text { Resistance mechanisms: XDR }(51 / 101) \text {, } \\
\text { PDR }(2 / 101) \text {, or MDR }(18 / 101) \\
\text { Other comments: ICU }(24 / 101) \\
\text { First line agent in } 39 / 101 \\
\text { Combination therapy }(36 / 101)\end{array}$ \\
\hline
\end{tabular}




\begin{tabular}{|c|c|c|c|}
\hline Article & Study type & Outcomes $^{1}$ & Comments \\
\hline & & $\begin{array}{l}\text { - Sepsis }(p=0.05) \text { and continuous renal } \\
\text { replacement therapy }(p=0.02) \\
\text { predicted clinical failure by multivariate } \\
\text { analysis }\end{array}$ & $\begin{array}{l}\text { Other agents used: aminoglycosides, } \\
\text { colistin, and carbapenems } \\
\text { Higher dose } 3 \text { g IV every } 8 \text { h used in } \\
\text { 25/101 (most HABP/VABP) }\end{array}$ \\
\hline $\begin{array}{l}\text { Hassan et al. } \\
\text { 2018. BMJ Case } \\
\text { Reports [36] }\end{array}$ & $\begin{array}{l}\text { Case report: } 62 \text {-year-old male with XDR } \\
\text { P. aeruginosa osteomyelitis upper } \\
\text { extremity with retention of } \\
\text { hardware }\end{array}$ & $\begin{array}{l}\text { - Successfully treated with } 8 \text { weeks } \mathrm{C} / \mathrm{T} \\
\text { - } 3 \text { months after completion, had } \\
\text { second open reduction internal fixation } \\
\text { with autograft placement which was } \\
\text { successful }\end{array}$ & $\begin{array}{l}\text { Infection type: hardware-associated OM } \\
\text { Organism: } P \text {. aeruginosa } \\
\text { Other comments: initial treatment: } \\
\text { ciprofloxacin }+ \text { cefepime } \times 8 \text { weeks with } \\
\text { hardware retained with recurrent } \\
\text { symptoms, loosening of hardware } \\
\text { requiring removal }\end{array}$ \\
\hline $\begin{array}{l}\text { Dietl et al. } 2018 . \\
\text { Int J } \\
\text { Antimicrobial } \\
\text { Agents [37] }\end{array}$ & $\begin{array}{l}\text { Retrospective observational study: } \mathrm{C} / \mathrm{T} \\
\text { for ABSSSI and osteomyelitis due to } \\
\text { XDR P. aeruginosa }\end{array}$ & $\begin{array}{l}\text { - Clinical cure 4/7 (57.1\%) } \\
\text { - No deaths }\end{array}$ & $\begin{array}{l}\text { Infection type: ABSSSI }(3 / 7) \text { and OM } \\
\quad(4 / 7) \\
\text { Organisms: XDR } P \text {. aeruginosa } \\
\text { Other comments: combination therapy } \\
(1 / 7) \\
\text { Other agents used: ampicillin }\end{array}$ \\
\hline $\begin{array}{l}\text { Haidar et al. } \\
\text { 2017. CID [38] }\end{array}$ & $\begin{array}{l}\text { Retrospective study: } \mathrm{C} / \mathrm{T} \text { for infections } \\
\text { due to MDR } P \text {. aeruginosa }\end{array}$ & $\begin{array}{l}\text { - Attributable } 30 \text {-day mortality } 1 / 21 \\
(4.2 \%) \\
\text { - Attributable } 90 \text {-day mortality } 4 / 21 \\
(19 \%) \\
\text { - } 15 / 21 \text { clinical cure }(71.4 \%)\end{array}$ & $\begin{array}{l}\text { Infection type: LRTI }(18 / 21) \text {, } \\
\quad \text { tracheobronchitis }(2 / 21) \text {, cIAI } \\
\quad(1 / 21) \text {, and cUTI }(1 / 21) \\
\text { Organisms: } P \text {. aeruginosa }(21 / 21) \\
\text { Polymicrobial }(6 / 21) \\
\text { Resistance mechanisms: (WGS done only } \\
\text { for } 6 \text { patients) } \\
\text { Amp-C }(6 / 21) \text { or OXA-48 }(6 / 21) \\
\text { Other comments: included } 9 / 21 \\
\text { immunocompromised }\end{array}$ \\
\hline $\begin{array}{l}\text { Popejoy et al. } \\
\text { 2017. BMC } \\
\text { Infectious } \\
\text { Diseases [39] }\end{array}$ & $\begin{array}{l}\text { Post hoc study from ASPECT cUTI and } \\
\text { CIAI phase III RCTs: outcomes in } \\
\text { patients with vs without DM }\end{array}$ & $\begin{array}{l}\text { - Clinical cure in cIAI } 75.4 \% \text { with DM vs } \\
86.1 \% \text { without DM }(p=0.0196) \\
\text { - Composite cure rate cUTI (mMITT) } 62.4 \\
\text { vs } 74.7 \%(p=0.1299) \\
\text { - Composite cure in patients without DM } \\
\text { with cUTI C/T } 79.5 \% \text { vs levofloxacin } \\
69.9 \%(p=0.048) \\
\text { - Patients with DM had more AEs ( } 49 \text { vs } \\
37.3 \%) \text { and serious AEs ( } 10.6 \text { vs } 4.6 \%)\end{array}$ & $\begin{array}{l}\text { Infection type: cIAI }(806 / 1606) \text { and } \\
\quad \text { CUTI (800/1606) } \\
\text { Organisms: E. coli }(1154 / 1606) \text {, } \\
\text { K. pneumoniae }(128 / 1606) \text {, } \\
\text { P. aeruginosa }(91 / 1606), \text { E. faecalis } \\
\text { (133/1606), E. faecium }(79 / 1606) \text {, and } \\
\text { anaerobes (390/1606) } \\
\text { Other comments: more comorbidities } \\
\text { and renal disease in patients with DM }\end{array}$ \\
\hline $\begin{array}{l}\text { Munita et al. } \\
\text { 2017. CID [40] }\end{array}$ & $\begin{array}{l}\text { Retrospective multicenter study: } \\
\text { outcomes of patients treated with } \\
\text { C/T for infections due to } \\
\text { carbapenem-resistant } P \text {. aeruginosa }\end{array}$ & $\begin{array}{l}\text { - Active against } 83 \% \text { P. aeruginosa } \\
\text { resistant to all other } \beta \text {-lactams } \\
\text { - Clinical success } 26 / 35(74.3 \%)\end{array}$ & $\begin{array}{l}\text { Infection type: RTI (18/35), BSI } \\
\quad(6 / 35)(1 \text { primary BSI), cIAI (5/35), } \\
\text { OM (5/35), LVAD (3/35), ABSSSI } \\
\quad(2 / 35) \text {, and cUTI }(1 / 35) \\
\text { Organisms: } P \text {. aeruginosa }(35 / 35) \\
\text { Other comments: combination therapy } \\
\text { (8/35) } \\
\text { Other agents used: tobramycin } \\
\text { (inhaled/IV), colistin (inhaled, IV), and } \\
\text { ciprofloxacin } \\
\text { Immunosuppressed }(10 / 35) \\
\text { Renal impairment }(13 / 35)\end{array}$ \\
\hline $\begin{array}{l}\text { Kullar et al. } \\
\text { 2017. J } \\
\text { Antimicrob } \\
\text { Chemother [41] }\end{array}$ & $\begin{array}{l}\text { Post hoc study from phase III RCTs } \\
\text { (ASPECT): clinical cure at TOC } \\
\text { (24-32 days from initiation) in } \\
\text { those with moderate RI (CrCl 30-50) } \\
\text { vs } \mathrm{CrCl}>50\end{array}$ & $\begin{array}{l}\text { - Higher risk treatment failure with } \\
\text { moderate RI compared to } \mathrm{CrCl}>50 \\
\text { for } \mathrm{cUTI}(\mathrm{RR}=2.39, p<0.001) \text { and } \\
\text { cIAI (RR }=3.32, p<0.001) \\
\text { regardless of treatment type } \\
\text { - cUTI with moderate RI clinical cure at } \\
\text { TOC (mMIT) } 25 / 31(81 \%) \mathrm{C} / \mathrm{T} \text { vs } 21 / 27 \\
\text { (78\%) levofloxacin } \\
\text { - cIAI with moderate RI } 11 / 23(48 \%) \\
\text { C/T vs } 9 / 13(69 \%) \text { meropenem }\end{array}$ & $\begin{array}{l}\text { Infection type: cIAI (806/1601) and } \\
\quad \text { cUTI ( } 795 / 1601) \\
\text { Other comments: disproportional } \\
\text { number of patients with renal } \\
\text { insufficiency in IAI group getting C/T }\end{array}$ \\
\hline $\begin{array}{l}\text { Castòn et al. } \\
2017 . \\
\text { Antimicrob } \\
\text { Agents } \\
\text { Chemother [42] }\end{array}$ & $\begin{array}{l}\text { Multicenter retrospective study in } \\
\text { Spain: C/T as salvage therapy for } \\
\text { MDR P. aeruginosa infections }\end{array}$ & $\begin{array}{l}\text { - All isolates sensitive } \\
\text { - Clinical success } 9 / 12(75 \%) \\
\text { - Microbiologic eradication 10/12 } \\
\text { (83.3\%) } \\
\text { - All-cause mortality } 3 / 12(25 \%)\end{array}$ & $\begin{array}{l}\text { Infection type: LRTI (6/12), cIAI } \\
\quad(4 / 12) \text {, BSI }(5 / 12) \text { (primary BSI } \\
\text { 1/12), and otitis/mastoiditis } \\
(1 / 12) \\
\text { Organisms: } P \text {. aeruginosa (12/12) } \\
\text { Other comments: } 10 / 12 \text { with septic } \\
\text { shock. Many comorbidities }\end{array}$ \\
\hline
\end{tabular}




\begin{tabular}{|c|c|c|c|}
\hline Article & Study type & Outcomes $^{1}$ & Comments \\
\hline $\begin{array}{l}\text { Popejoy et al. } \\
\text { 2017. J } \\
\text { Antimicrob } \\
\text { Chemother } \\
{[43 \bullet]}\end{array}$ & $\begin{array}{l}\text { Pooled data from ASPECT cUTI and cIAI } \\
\text { phase III RCTs: outcomes in } \\
\text { patients with cUTI or cIAI due to } \\
\text { ESBL Enterobacteriaceae }\end{array}$ & $\begin{array}{l}\text { - Clinical cure at TOC (5-9 days } \\
\text { post-EOT for UTI, } 24-32 \text { days } \\
\text { post-EOT for cIAI) from pooled ME } \\
76 / 78(97.4 \%) \text { C } / T \text { vs } 38 / 46 \\
(82.6 \%) \text { levofloxacin for cUTI ( } p= \\
0.011) \text { and } 23 / 26(88.5 \%) \\
\text { meropenem for cIAI }(p>0.05) \\
\text { - Microbiologic cure at TOC } 62 / 78 \\
(79.5 \%) \text { C/T vs } 45 / 72(62.5 \%) \\
\text { comparators }(p=0.022)\end{array}$ & $\begin{array}{l}\text { Infection type: cUTI }(100 / 150) \text { and } \\
\quad \text { cIAI ( } 50 / 150) \\
\text { Organisms: mostly E. coli }(103 / 159) \text { and } \\
\text { K. pneumoniae (28/159) } \\
\text { Resistance mechanisms: CTX-M } \\
(107 / 150), \text { OXA-1/30 (30/150), SHV, } \\
\text { and/or TEM (37/150). Other ESBL } \\
\text { (7/150) }\end{array}$ \\
\hline $\begin{array}{l}\text { Miller et al. } 2016 . \\
\text { Antimicrob } \\
\text { Agents } \\
\text { Chemotherapy } \\
\text { [44] }\end{array}$ & $\begin{array}{l}\text { Data from ASPECT cIAI phase III RCT: } \\
\text { clinical and microbiologic outcomes } \\
\text { in patients with cIAI due to } \\
\text { P. aeruginosa }\end{array}$ & $\begin{array}{l}\text { Clinical and microbiologic cure in } \\
26 / 26(100 \%) \text { C/T vs } 27 / 29 \\
(93.1 \%) \text { meropenem }\end{array}$ & $\begin{array}{l}\text { Infection type: cIAI }(72 / 72) \text { and BSI } \\
\quad(3 / 72) \\
\text { Organisms: P. aeruginosa (72/72) } \\
\text { Polymicrobial ( } 68 / 72) \\
\text { Resistance mechanisms: MDR (3/72) and } \\
\text { amp-C (11/72) }\end{array}$ \\
\hline $\begin{array}{l}\text { Huntington et al. } \\
2016 . \mathrm{J} \\
\text { Antimicrob } \\
\text { Agents } \\
\text { Chemother [45] }\end{array}$ & $\begin{array}{l}\text { Data from ASPECT cUTI phase III RCT: } \\
\text { post hoc analysis to evaluate } \\
\text { efficacy of C/T vs levofloxacin in } \\
\text { levofloxacin-resistant isolates }\end{array}$ & $\begin{array}{l}\text { - Clinical cure rates in mMITT C/T } \\
60 / 100(60 \%) \text { vs levofloxacin, } \\
44 / 112(39.3 \%) \\
\text { - ESBL clinical cure } 28 / 48(58.3 \%) \text { C/T vs } \\
15 / 42(35.7 \%) \text { levofloxacin }\end{array}$ & $\begin{array}{l}\text { Infection type: cUTI } \\
\text { Organisms: mostly E. coli (144/212), } \\
\text { K. pneumoniae (26/212), and } \\
P \text {. aeruginosa (12/212) } \\
\text { Resistance mechanisms: ESBL (85/212), } \\
\text { CTX-M-14/15 (69/212), and/or ST131 } \\
(37 / 212)\end{array}$ \\
\hline $\begin{array}{l}\text { Wagenlehner } \\
\text { et al. 2015. } \\
\text { Lancet }[\mathbf{4 6 \bullet \bullet ] . ~}\end{array}$ & $\begin{array}{l}\text { Phase III double-blind multicenter } \\
\text { international RCT (ASPECT cUTI): } \\
\text { C/T vs levofloxacin for cUTI } \\
\text { including pyelonephritis }\end{array}$ & $\begin{array}{l}\text { - C/T noninferior and superior for } \\
\text { composite cure at TOC (5-9 days } \\
\text { post-therapy) in mMITT } 306 / 398 \\
(76.9 \%) \text { C/T vs } 275 / 402(68.4 \%) \\
\text { levofloxacin } \\
\text { - Noninferiority and superiority for } \\
\text { microbiologic eradication in mMITT } \\
320 / 398(80.4 \%) \text { vs } 290 / 402(72.1 \%) \\
\text { - Higher composite cure rates in } \\
\text { subpopulations }>65, \text { cUTI, } \\
\text { levofloxacin-resistant and ESBL }\end{array}$ & $\begin{array}{l}\text { Infection type: cUTI and acute } \\
\quad \text { pyelonephritis } \\
\text { BSI }(72 / 800) \\
\text { Organisms: mostly E. coli }(629 / 800) \text {, } \\
\text { K. pneumoniae (58/800), P. mirabilis } \\
(24 / 800) \text {, and P. aeruginosa }(23 / 800) \\
\text { Polymicrobial ( } 24 / 800) \\
\text { Resistance mechanisms: ESBL }(118 / 800)\end{array}$ \\
\hline $\begin{array}{l}\text { Solomkin et al. } \\
\text { 2015. CID } \\
{[47 \bullet \bullet]}\end{array}$ & $\begin{array}{l}\text { Phase III double-blind multicenter RCT } \\
\text { (ASPECT cIAI): } C / T+\text { metronidazole } \\
\text { vs meropenem for clinical cure of } \\
\text { CIAI }\end{array}$ & $\begin{array}{l}\text { - Noninferior for clinical cure at TOC } \\
(24-32 \text { days after start of therapy) } \\
323 / 389(83 \%) \text { vs } 364 / 417(87.3 \%) \\
\text { meropenem } \\
\text { - Clinical cure in ESBL } 23 / 24(95.8 \%) \text { vs } \\
23 / 36(88.5 \%) \\
\text { - All-cause mortality } 11 / 482(8.1 \%) \text { vs } \\
36 / 427(7.2 \%)\end{array}$ & $\begin{array}{l}\text { Infection type: cIAI } \\
\text { Organisms: mostly E. coli }(525 / 806) \text {, } \\
\text { K. pneumoniae }(76 / 806) \text {, and } \\
\text { P. aeruginosa (72/806) } \\
\text { Polymicrobial ( } 545 / 806) \\
\text { Resistance mechanisms: ESBL }(58 / 806) \\
\text { or CTX-M (13/806). MDR P. aeruginosa } \\
\text { (9/806) } \\
\text { Other comments: } \\
\text { AE: mostly GI (44\%), C. difficile infection } \\
\text { in } 1 \text { patient from each group }\end{array}$ \\
\hline $\begin{array}{l}\text { Lucasti et al. } \\
2014 . \\
\text { Antimicrob } \\
\text { Agents } \\
\text { Chemother [48] }\end{array}$ & $\begin{array}{l}\text { Phase II double-blind multicenter RCT: } \\
\text { C/T + metronidazole vs meropenem } \\
\text { for cIAI }\end{array}$ & $\begin{array}{l}\text { - Clinical cure at TOC (7-14 days after } \\
\text { EOT) } 51 / 61(83.6 \%) \text { vs } 24 / 25(96 \%) \\
\text { in mMITT } \\
\text { - Microbiologic cure } 48 / 53(90.6 \%) \text { vs } \\
23 / 24(95.8 \%)\end{array}$ & $\begin{array}{l}\text { Infection type: cIAI } \\
\text { Organisms: mostly E. coli }(60 / 86) \text {, } \\
\text { Streptococcus spp., }(8 / 86) \text {, } \\
\text { K. pneumoniae }(8 / 86), \text { and } E \text {. faecium } \\
\text { (8/86) } \\
\text { Polymicrobial }(33 / 86) \\
\text { Resistance mechanisms: ESBL }(3 / 86) \\
\text { Other comments: most common AE: } \\
\text { pyrexia and GI }\end{array}$ \\
\hline \multicolumn{4}{|l|}{ Delafloxacin } \\
\hline $\begin{array}{l}\text { Pullman et al. } \\
\text { 2017. J } \\
\text { Antimicrob } \\
\text { Chemo }[49 \bullet \bullet]\end{array}$ & $\begin{array}{l}\text { Phase III double-blind multicenter } \\
\text { international noninferiority RCT: } \\
\text { delafloxacin vs } \\
\text { vancomycin/aztreonam for ABSSSI }\end{array}$ & $\begin{array}{l}\text { - Noninferior for objective clinical } \\
\text { response ( } \geq 20 \% \text { erythema } \\
\text { reduction) at } 48-72 \text { h } 259 / 331 \\
\text { ( } 78.2 \%) \text { vs } 266 / 329(80.9 \%) \\
\text { (investigator assessed cure and } \\
\text { success) } \\
\text { - Microbiologic eradication: } 175 / 179 \\
(97.8 \%) \text { delafloxacin vs } 181 / 184 \\
\text { (98.4\%) vancomycin/ aztreonam } \\
\text { - Noninferior in MRSA subgroup }\end{array}$ & $\begin{array}{l}\text { Infection type: ABSSSI. BSI }(15 / 660) \\
\text { Organisms: mostly MRSA }(169 / 660) \text {, } \\
\text { MSSA (156/660), S. anginosus }(67 / 660) \text {, } \\
\text { S. epidermidis }(33 / 660), \text { K. pneumoniae } \\
(21 / 660), \text { E. coli }(14 / 660), \text { S. pyogenes } \\
(12 / 660) \text {, and S. lugdunensis }(10 / 66) \\
\text { Other comments: } \\
\text { AE: } 47.5 \% \text { delafloxacin-majority } \\
\text { mild/GI }\end{array}$ \\
\hline
\end{tabular}


Table 2. (Continued)

Article

Study type

Kingsley et al. 2016. J

Antimicrob

Chemother

[50•]

0 'Riordan et al. 2015. Int J Infectious Diseases [51]

Eravacycline

Solomkin et al. 2017. JAMA

Surgery $[52 \bullet \bullet]$

International multicenter double-blind noninferiority RCT (IGNITE 1): eravacycline vs ertapenem $1 \mathrm{~g}$ every $24 \mathrm{~h}$ in CIAI

Phase II double-blind multicenter RCT: comparing 2 doses with tigecycline for complicated ABSSSI

Double-blind phase II RCT: delafloxacin vs linezolid and vancomycin for treatment of ABSSSI

Solomkin et al.

2014.

Antimicrob

Agents

Chemother [53]
Phase II double-blind multicenter international RCT: eravacycline at 2 doses $(1.5 \mathrm{mg} / \mathrm{kg}$ vs $1.0 \mathrm{mg} / \mathrm{kg})$ compared to ertapenem $1 \mathrm{~g}$ every $24 \mathrm{~h}$ IV for efficacy in cIAI
Imipenem/cilastatin (IMI) + relebactam (REL)

Lucasti et al. 2016.

Antimicrob Agents

Chemother [54]
Outcomes $^{1}$

- Investigator assessed cure at late

follow-up (days 21-28) in obese subgroup 86/120 (71.7\%) delafloxacin vs $54 / 95(57.4 \%)$

vancomycin/aztreonam

- Investigator-assessed clinical cure $57 / 81(70.4 \%)$ delafloxacin vs $50 / 77(64.9 \%)$ linezolid $(p=0.496)$ vs $53 / 98(54.1 \%)$ vancomycin $(p=$ $0.031)$

- Clinical cure in obese patients with delafloxacin $26 / 33(78.8 \%)$ vs

vancomycin $20 / 41(48.8 \%)(p=0.009)$

- No difference in clinical cure for MRSA

$(19 / 29(65.5 \%)$ vs $21 / 34(61.8 \%)$ vs

$21 / 32(65.6 \%)$ or for different types of infection

- Comparable to tigecycline for treatment of complicated ABSSSI

- Clinical cure at TOC (14-21 days after EOT) (CE) delafloxacin $300 \mathrm{mg}$ every 12 h 33/35 (94.3\%) vs $450 \mathrm{mg}$ every $12 \mathrm{~h} 37 / 40$ (92.5\%) vs tigecycline $31 / 14(91.2 \%)$

- Comparable clinical cure at TOC in subgroup analysis for $S$. aureus $21 / 22$ $(95.5 \%)$ vs $25 / 27(92.6 \%)$ vs $18 / 20$ $(90 \%)$ and MRSA $13 / 14(92.9 \%)$ vs $19 / 20(95 \%)$ vs $12 / 14(85.7 \%)$

- Noninferiority met for composite clinical cure $235 / 270(87 \%)$ eravacycline vs $238 / 268(88.8 \%)$ ertapenem at TOC (25-31 days after first dose)

- Noninferiority met for clinical cure in mMITT analysis at TOC $191 / 220(86.8 \%)$ eravacycline vs $198 / 226(87.6 \%)$

- All-cause mortality $3 / 268(1.1 \%)$

eravacycline vs $6 / 270(2.2 \%)$ ertapenem

- Clinical response at TOC (10-14 days after first dose) in ME 39/42 $(92.9 \%)$ high dose eravacycline vs 41/41 (100\%) low-dose eravacycline vs $24 / 26(92.3 \%)$ ertapenem

- Clinical cure at TOC in ESBL 8/10 (80\%) high dose vs $10 / 10(100 \%)$ low dose vs $4 / 4(100 \%)$ ertapenem

- Microbiologic response at TOC 39/42 $(92.9 \%)$ high-dose eravacycline, $41 / 41$ $(100 \%)$ low-dose eravacycline vs $24 / 26$ (92.3\%) ertapenem

- All-cause mortality $3 / 42$ (7.1\%)

high-dose ertapenem, 0 in other groups

- Both doses REL + IMI noninferior to IMI alone for clinical response at discontinuation of IV therapy in $\mathrm{ME}^{6}$ $78 / 81(96.3 \%) 250 \mathrm{mg} \mathrm{REL}+$ IMI vs

\section{Comments}

Infection type: ABSSSI

Organisms: mostly MRSA (106/256) and MSSA (53/256) identified

AE: nausea, vomiting, diarrhea, liver toxicity

Infection type: complicated ABSSSI Organisms: mostly MRSA (68/150) and MSSA (28/150)

Infection type: cIAI

Organisms: mostly E. coli (259/446), anaerobes (213/446), Streptococcus spp. $(129 / 446)$, E. faecalis (49/446), E. faecium (46/446), K. pneumoniae (41/446), P. aeruginosa (38/446), P. mirabilis (25/446), and Acinetobacter spp. $(14 / 446)$

Resistance mechanisms: ESBL (35/446) and carbapenemase $(7 / 446)$

Other comments: excluded RI and liver disease

AE: eravacycline $113 / 270$ (41.9\%) vs ertapenem 75/268 (26.9\%), 13 life-threatening in each group. More nausea and phlebitis in eravacycline

Infection type: cIAI

Organisms: mostly E. coli (86/144) and K. pneumoniae $(14 / 144)$

Resistance mechanisms: ESBL (36/144)

Other comments: highest MIC for eravacycline was $P$. aeruginosa

(4-16 $\mu \mathrm{g} / \mathrm{mL})$; otherwise, highest was $2 \mu \mathrm{g} / \mathrm{mL}$

AE: $35.8 \%$. Most mild and GI

Infection type: cIAI. BSI (10/277)

Organisms: mostly E. coli $(162 / 250)$, $P$. aeruginosa $(36 / 250)$, K. pneumoniae (34/250), P. mirabilis (18/250), 


\begin{tabular}{|c|c|c|c|}
\hline Article & Study type & Outcomes $^{1}$ & Comments \\
\hline & & $\begin{array}{l}85 / 86(98.8 \%) 125 \mathrm{mg} \text { REL + IMI vs } \\
79 / 83(95.2 \%) \text { IMI alone } \\
(p<0.001) \\
\text { - Clinical response at short-term } \\
\text { follow-up (5-9 days after EOT) } 75 / 79 \\
(94.9 \%) 250 \mathrm{mg} \text { REL + IMI vs } 81 / 86 \\
(94.2 \%) 125 \mathrm{mg} \text { REL + IMI vs } 78 / 81 \\
(96.3 \%) \text { IMI alone } \\
\text { - Clinical response at long-term } \\
\text { follow-up (28 days after EOT) } 74 / 79 \\
(93.7 \%) 250 \mathrm{mg} \text { REL + IMI vs } 81 / 85 \\
(95.3 \%) 125 \mathrm{mg} \text { REL + IMI vs } 75 / 79 \\
(94.9 \%) \text { IMI alone in ME } \\
\text { - } 34 / 34(100 \%) \text { (ME) IMI-NS isolates } \\
\text { with clinical response at EOT } \\
\text { - } 500 \mathrm{mg} \text { IMI + } 250 \mathrm{mg} \text { REL covered }> \\
90 \% \text { carbapenem resistant bacteria }\end{array}$ & $\begin{array}{l}\text { S anginosus (18/250), E. faecalis } \\
(17 / 250), \text { E. cloacae }(15 / 250) \text {, and } \\
\text { anaerobes }(81 / 250) \\
\text { Other comments: only } 10 \text { with APACHE }> \\
15 \text {. Stratified by disease severity } \\
\text { Excluded } \mathrm{CrCl}<50 \text {, hepatic dysfunction, } \\
\text { and APACHE }>30 \\
\text { AE: } 45.8 \% \text { (GI most common) } \\
\text { Serious AE: severe thrombocytosis with } \\
\text { IMI alone; reduced CrCl; } 3 \text { with IMI alone } \\
\text { had thrombocytosis, nausea, increased } \\
\text { ALT }\end{array}$ \\
\hline \multicolumn{4}{|l|}{ Lefamulin (BC-3781) } \\
\hline $\begin{array}{l}\text { Prince et al. } 2013 . \\
\text { Antimicrob } \\
\text { Agents } \\
\text { Chemother [55] }\end{array}$ & $\begin{array}{l}\text { Double-blind multicenter phase II RCT: } \\
\text { BC-3781 in ABSSSI caused by } \\
\text { gram-positive pathogen vs } \\
\text { vancomycin } 1 \mathrm{~g} \text { IV every } 12 \mathrm{~h}\end{array}$ & $\begin{array}{l}\text { - Clinical success at TOC (7-14 days } \\
\text { post-treatment) in CE } 54 / 60(90 \%) \\
100 \mathrm{mg} \text { vs } 48 / 54(88.9 \%) 150 \mathrm{mg} \\
\text { vs } 51 / 47(92.2 \%) \text { vancomycin } \\
\text { - Clinical success at TOC in mMITT } 41 / 50 \\
(82 \%) 100 \mathrm{mg} \text { vs } 42 / 51(82 \%) 150 \mathrm{mg} \\
\text { vs } 42 / 51(82 \%) \text { vancomycin } \\
\text { - Comparable clinical success in } \\
\text { subgroup analyses for baseline } \\
\text { pathogen, primary infection type, DM } \\
\text { status } \\
\text { - Microbiologic success at TOC: } 40 / 50 \\
(80 \%) 100 \text { mg vs } 43 / 51(84.3 \%) \\
150 \text { mg vs } 42 / 51 \text { ( } 82.4 \%) \text { vancomycin } \\
\text { - Similar decrease in lesion size, time to } \\
\text { cessation in spread in erythema, clinical } \\
\text { response at day } 3\end{array}$ & $\begin{array}{l}\text { Infection type: ABSSSI } \\
\text { Organisms: mostly MRSA (105/152) and } \\
\text { MSSA (34/152) }\end{array}$ \\
\hline \multicolumn{4}{|c|}{ Meropenem/vaborbactam (M-V) } \\
\hline $\begin{array}{l}\text { Wunderink et al. } \\
\text { 2018. Infect Dis } \\
\text { Ther [56・日 }\end{array}$ & $\begin{array}{l}\text { Open-label multicenter phase III RCT } \\
\text { (TANGO II): efficacy and safety of } \\
\text { M-V vs best-available therapy for } \\
\text { CRE infections }\end{array}$ & $\begin{array}{l}\text { - Clinical cure at EOT } 21 / 32(65.6 \%) \\
\text { M-V vs } 5 / 15(33.3 \%) \text { BAT }(p=0.03) \\
\text { - Clinical cure at TOC (7 days after EOT) } \\
\text { in mCRE mIT' } 19 / 32(59.4 \%) \text { M-V vs } \\
4 / 15(26.7 \%) \text { BAT ( } p=0.02) \\
\text { - Microbiologic cure at EOT mCRE mITT } \\
21 / 32(65.6 \%) \text { M-V vs } 6 / 15(40 \%) \text { BAT } \\
(p=0.09) \\
\text { - Microbiologic cure in mCRE mITT at TOC } \\
17 / 32(53.1 \%) \text { M-V vs } 5 / 15(33.3 \%) \text { BAT } \\
(p=0.19) \\
\text { - All-cause mortality at } 28 \text { days mCRE } \\
\text { mIT } 5 / 32(15.6 \%) \text { M-V vs } 5 / 15(33.3 \%) \\
\text { BAT ( } p=0.20) \\
\text { - Clinical cure in immunocompromised } \\
7 / 11(63.6 \%) \text { M-V vs } 0 / 8 \text { BAT ( } 0 \%) \\
(p<0.001) \\
\text { - All-cause mortality in patients without } \\
\text { prior antibiotic failure M-V } 1 / 23(4.3 \%) \\
\text { vs BAT } 5 / 15(33.3 \%)(p=0.02) \\
\text { - Clinical cure at TOC in patients with } \\
\text { prior antibiotic failure M-V } 16 / 23 \\
\text { (69.6\%) vs BAT } 4 / 15 \text { ( } 26.7 \%)(p= \\
0.004) \\
\text { - No statistical difference in clinical cure } \\
\text { by infection subtypes }\end{array}$ & $\begin{array}{l}\text { Infection type: BSI (22/47), CUTI } \\
\quad(16 / 47), \text { HABP/VABP }(5 / 47) \text {, and } \\
\quad \text { CIAI (4/47) } \\
\text { Organisms: mostly } K . \text { pneumoniae } \\
\text { (41/47) } \\
\text { Resistance mechanisms: CRE (37/47), } \\
\text { MBL (0XA-48 or NDM1) }(4 / 47) \text {, or KPC } \\
\text { (1/47) } \\
\text { Other comments: } \\
\text { BAT used: polymixins, carbapenems, } \\
\text { aminoglycosides, tigecycline, and CZA } \\
\text { More patients with prior antibiotic } \\
\text { failure in M-V group } \\
\text { High amount of RI, comorbidities, sepsis } \\
\text { Immunocompromised } 19 / 47 \\
\text { Fewer AE in M-V, including less renal } \\
\text { toxicity ( } 42 / 50 \text { vs } 23 / 25)\end{array}$ \\
\hline $\begin{array}{l}\text { Hanretty et al. } \\
2018 .\end{array}$ & Pharmacotherapy [57] & $\begin{array}{l}\text { Case report: } M-V \text { in } 4 \text {-year-old male } \\
\text { with KPC } K \text { pneumoniae bacteremia }\end{array}$ & $\begin{array}{l}\text { - Clinical and microbiologic cure } \\
\text { obtained } \\
\text { - Dose } 40 \mathrm{mg} / \mathrm{kg} \text { IV every } 6 \mathrm{~h} \text { over } 3 \mathrm{~h} \\
\text { had successful target attainment }\end{array}$ \\
\hline
\end{tabular}




\begin{tabular}{|c|c|c|c|}
\hline Article & Study type & Outcomes $^{1}$ & Comments \\
\hline $\begin{array}{l}\text { Kaye et al. } 2018 . \\
\text { JAMA }[58 \bullet \bullet]\end{array}$ & $\begin{array}{l}\text { Phase III double-blind multicenter } \\
\text { noninferiority (TANGO I) RCT: } M-V \text { vs } \\
P / T \text { in CUTI }\end{array}$ & $\begin{array}{l}\text { - Noninferiority met with superiority } \\
\text { for overall success (clinical cure or } \\
\text { improvement with eradication) in } \\
\text { mMITT at EOT } 189 / 192(98.4 \%) \mathrm{M}-\mathrm{V} \\
\text { vs } 171 / 182(94 \%) \mathrm{P} / \mathrm{T}(p<0.001) \\
\text { - Clinical cure in mMITT at TOC } 174 / 192 \\
\text { (90.6\%) vs } 157 / 182(86.3 \%) \\
\text { - Noninferiority maintained when } \\
\text { accounting for center effect and } \\
\text { geographic region } \\
\text { - Microbiologic cure at TOC (7 days after } \\
\text { EOT) by FDA criteria in mMITT } 132 / 192 \\
\text { (68.8\%) M-V vs } 113 / 182(62.1 \%) \mathrm{P} / \mathrm{T} \\
\text { ( } p<0.001) \\
\text { - Similar results for cUTI vs acute } \\
\text { pyelonephritis }\end{array}$ & $\begin{array}{l}\text { Infection type: cUTI and acute } \\
\text { pyelonephritis } \\
\text { Organisms: mostly E. coli (242/374), } \\
\text { K. pneumoniae (58/374), E. faecalis } \\
(27 / 374), \text { P mirabilis }(18 / 374) \text {, and } \\
\text { E. cloacae complex }(15 / 374) \\
\text { Other comments: AE similar across } \\
\text { groups-headache most common }\end{array}$ \\
\hline \multicolumn{4}{|l|}{ Plazomicin } \\
\hline $\begin{array}{l}\text { Connolly et al. } \\
2018 . \\
\text { Antimicrobial } \\
\text { Agents and } \\
\text { Chemotherapy } \\
{[59 \bullet]}\end{array}$ & $\begin{array}{l}\text { Phase II multicenter double-blind RCT: } \\
\text { efficacy and safety of plazomicin vs } \\
\text { levofloxacin for cUTI and acute } \\
\text { pyelonephritis }\end{array}$ & $\begin{array}{l}\text { - Microbiologic eradication rates at } \\
\text { TOC (5-12 days post-EOT) } 6 / 12 \\
(50 \%) 10 \mathrm{mg} / \mathrm{kg} \text { plazomicin vs } \\
31 / 51(60.8 \%) 15 \mathrm{mg} / \mathrm{kg} \\
\text { plazomicin vs } 17 / 29(58.6 \%) \\
\text { levofloxacin } \\
\text { - Clinical cure at TOC: } 8 / 12(66.7 \%) \\
10 \mathrm{mg} / \mathrm{kg}, 36 / 51(70.6 \%) 15 \mathrm{mg} / \mathrm{kg} \text {, } \\
19 / 29(65.5 \%) \text { levofloxacin } \\
\text { - Rise in serum } \mathrm{Cr} \text { by at least } 0.5 \mathrm{mg} / \mathrm{dL} \\
\text { during the study } 1 / 22(4.5 \%) 10 \mathrm{mg} / \mathrm{kg} \\
\text { vs } 4 / 74(5.6 \%) 15 \mathrm{mg} / \mathrm{kg} \text { vs } 1 / 41(2.4 \% \\
\text { levofloxacin) }\end{array}$ & $\begin{array}{l}\text { Infection type: cUTI and acute } \\
\text { pyelonephritis } \\
\text { Organisms: mostly } E \text {. coli }(45 / 63) \\
\text { Other comments: } \\
\text { AE: } 7 / 2210 \mathrm{mg} / \mathrm{kg}, 26 / 7415 \mathrm{mg} / \mathrm{kg} \text {, } \\
21 / 44 \text { levofloxacin most frequent } \\
\text { headache, nausea, vomiting, diarrhea, } \\
\text { dizziness; } 2 / 7415 \mathrm{mg} / \mathrm{kg} \text { with } \\
\text { vestibular/cochlear AE }\end{array}$ \\
\hline \multicolumn{4}{|l|}{ Solithromycin } \\
\hline $\begin{array}{l}\text { File et al. } 2016 . \\
\text { CID }[60 \bullet \bullet]\end{array}$ & $\begin{array}{l}\text { Phase III international multicenter } \\
\text { noninferiority RCT (SOLITAIRE-IV): } \\
\text { IV to PO solithromycin vs } \\
\text { moxifloxacin for CABP }\end{array}$ & $\begin{array}{l}\text { - Noninferior to moxifloxacin at early } \\
\text { clinical response ( } 72 \mathrm{~h} \\
\text { post-initiation of therapy) } 344 / 434 \\
(79.3 \%) \text { solithromycin vs } 342 / 429 \\
\text { (79.7\%) moxifloxacin and } \\
\text { short-term follow-up (5-10 days } \\
\text { post-E0T) } 367 / 434(84.6 \%) \\
\text { solithromycin vs } 380 / 429(88.6 \%) \\
\text { moxifloxacin } \\
\text { - Noninferiority maintained in subgroup } \\
\text { analysis of PORT, mMITT } \\
\text { - All-cause mortality } 5 / 434(1.2 \%) \\
\text { solithromycin vs } 7 / 429 \text { moxifloxacin } \\
\text { (1.6\%) }\end{array}$ & $\begin{array}{l}\text { Infection type: CABP, BSI }(22 / 326) \\
\text { Organisms: mostly S. pneumoniae } \\
(155 / 326), \text { M. pneumoniae }(69 / 326) \\
\text { S. aureus }(37 / 326 \text {, including } 3 \mathrm{MRSA}) \text {, } \\
\text { H. influenzae }(38 / 326) \text {, Legionella spp. } \\
\text { (36/326), K. pneumoniae }(12 / 326) \text {, and } \\
\text { P. aeruginosa (10/326) } \\
\text { Other comments: excluded reduced CrCl. } \\
\text { Given } 800 \mathrm{mg} \text { IV } \times 1 \text { followed by } 400 \mathrm{mg} \\
\text { PO days } 2-5\end{array}$ \\
\hline $\begin{array}{l}\text { Barrera et al. } \\
\text { 2016. Lancet } \\
\text { Infect Dis } \\
{[61 \bullet \bullet]}\end{array}$ & $\begin{array}{l}\text { Double-blind international multicenter } \\
\text { phase III noninferiority RCT } \\
\text { (SOLITAIRE-oral): solithromycin PO } \\
\text { vs moxifloxacin for CABP }\end{array}$ & $\begin{array}{l}\text { - Noninferior to moxifloxacin } \times 7 \text { days } \\
\text { at early clinical response }(72 \mathrm{~h} \\
\text { post-initiation of therapy) } 333 / 426 \\
(78.2 \%) \text { vs } 338 / 434(77.9 \%) \\
\text { moxifloxacin, short-term and } \\
\text { long-term follow-up } \\
\text { - Similar results based on subgroups (> } \\
65, \text { hx COPD/asthma) }\end{array}$ & $\begin{array}{l}\text { Infection type: CABP } \\
\text { Organisms: mostly S. pneumoniae (198), } \\
\text { H. influenzae (135), M. pneumoniae } \\
\text { (79), Legionella spp. (125), and } \\
\text { Moraxella spp. (51) } \\
\text { Other comments: stratified by PORT } \\
\text { score, COPD, asthma } \\
\text { AE: } 155 / 424(36.6 \%) \text { solithromycin vs } \\
\text { 154/432 (35.6\%) moxifloxacin. Most GI, } \\
\text { headache, dizziness }\end{array}$ \\
\hline $\begin{array}{l}\text { Hook et al. } 2015 . \\
\text { CID [62] }\end{array}$ & $\begin{array}{l}\text { Open-label two-center phase II RCT: } \\
1200 \mathrm{mg} \text { vs } 1000 \mathrm{mg} \text { dose efficacy } \\
\text { for } \mathrm{N} \text {. gonorrhea based on test of } \\
\text { cure culture results (7-10 days after } \\
\text { administration) }\end{array}$ & $\begin{array}{l}\text { - Culture remained negative for } \\
N \text {. gonorrhea at } 7-10 \text { days for all } \\
\text { sites tested in both groups } \\
\text { - NAAT remained positive at } 1 \text { week in } \\
5 / 411200 \mathrm{mg}(12.2 \%) \text { and } 5 / 37(13.5 \%) \\
1000 \mathrm{mg} \text { of those positive prior to } \\
\text { treatment } \\
\text { - C. trachomatis low prevalence (19\%), } \\
\text { cleared at } 1 \text { week in } 7 / 8(87.5 \%) \\
1200 \text { mg and } 2 / 3(66.7 \%) 1000 \mathrm{mg}\end{array}$ & $\begin{array}{l}\text { Infection type: GU }(59 / 59) \text {, } \\
\text { pharyngeal ( } 8 / 59 \text { or } 16 / 59 \text { on } \\
\text { NAAT), and rectal ( } 4 / 59 \text { or } 20 / 59 \text { on } \\
\text { NAAT) } \\
\text { Other comments: low prevalence of } \\
\text { co-infection } \\
\text { AE: self-limited diarrhea, nausea }\end{array}$ \\
\hline
\end{tabular}




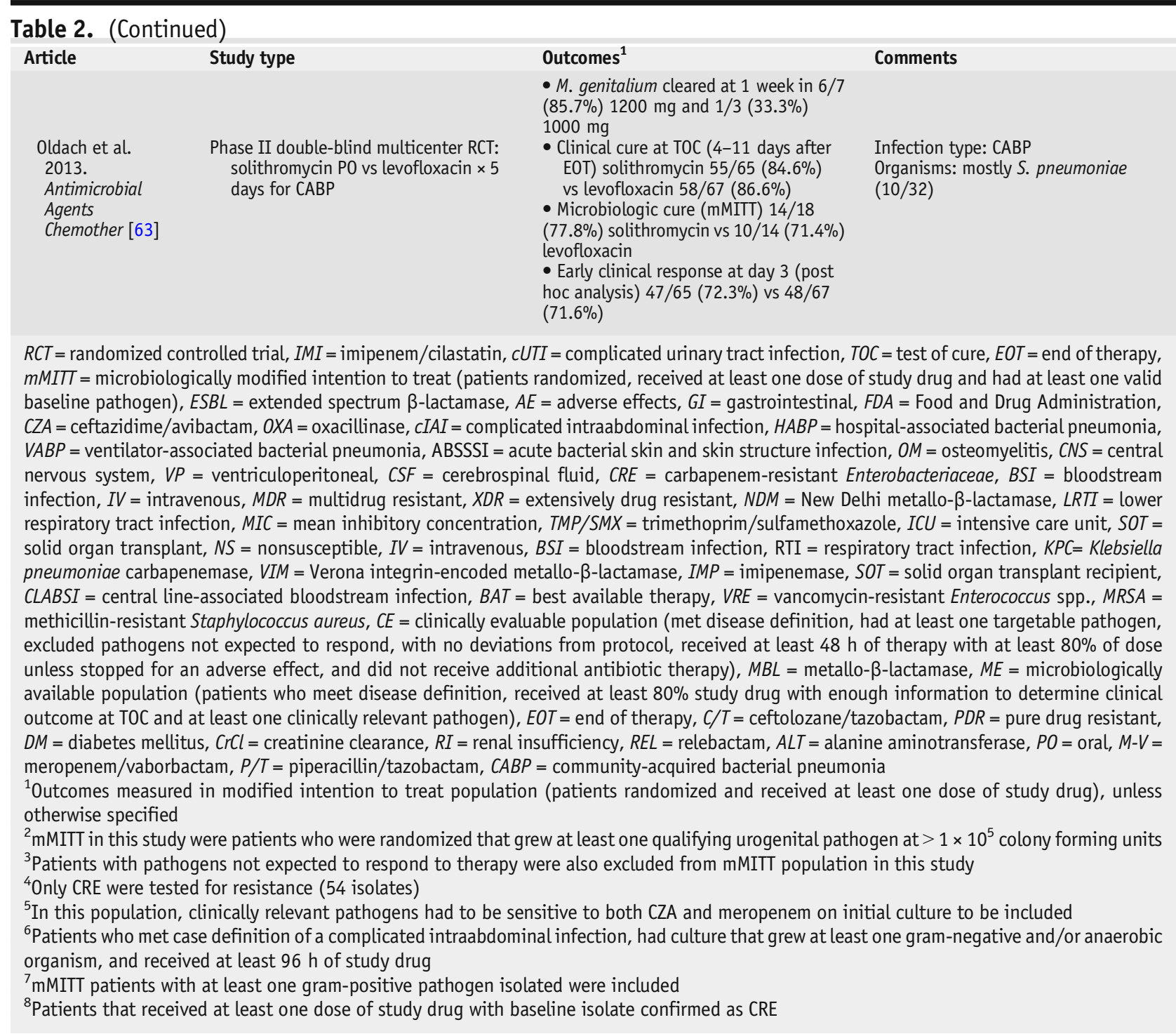

dysfunction. CZA is not associated with QTc prolongation [72, 73].

CZA was initially FDA approved for treatment of complicated intraabdominal infections (CIAI) in combination with metronidazole and cUTI


$29 \bullet \bullet, 74]$. The RECLAIM trials demonstrated noninferiority to meropenem for


maintained efficacy in patients with isolates that screened positive for ESBL in these trials $[24 \bullet, 30]$. The RECAPTURE trials determined noninferiority to

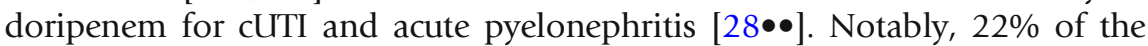
isolates recovered in these studies were ceftazidime nonsusceptible, and a limited number of critically ill patients were included. The REPRISE trial demonstrated the efficacy of CZA for treating CUTI and CIAI due to ceftazidime-nonsusceptible isolates [27•]. The REPROVE trial by Torres et al. demonstrated noninferiority to meropenem in the treatment of HABP and VABP, prompting 


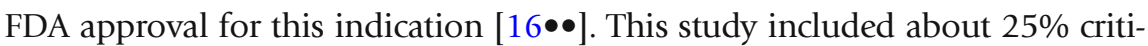

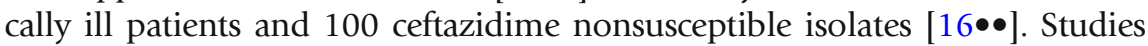
investigating the efficacy of CZA in infections due to MDR organisms and CRE are limited by small sample size or observational study design. Observational studies demonstrate success in treating KPC K. pneumoniae, including a prospective study by Van Duin et al. that demonstrated superiority to colistin with $64 \%$ better outcomes overall $[10,17 \bullet, 20]$. In small observational studies using CZA for the treatment of CRE infections, mostly due to KPC or OXA-48 producing $K$. pneumoniae, the clinical success ranges from 50 up to $86 \%$ with up to $30 \%$ mortality and significantly worse outcomes in patients requiring continuous renal replacement therapy or with higher severity of illness $[10,12 \bullet, 13,20,21$, $23,26]$. It has poor activity against isolates expressing NDM-1, VIM-2, OXA-23, or PER-1 and about $50-70 \%$ success in the treatment of MDR or extensive drug-

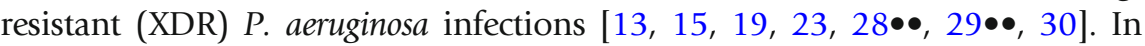
pooled isolates from five RCTs, there were 89.7 and $66.1 \%$ sensitivity to MDR Enterobacter cloacae $(n=29)$ and $P$. aeruginosa $(n=56)$, respectively, and a minimal decrease in MICs compared to ceftazidime alone for $P$. aeruginosa [75]. Case reports note success in using CZA to treat other types of infections, including ventriculitis and hardware-associated osteomyelitis, due to KPC and MBL producing $K$. pneumoniae or $P$. aeruginosa $[11,14,18]$. There are ongoing phase II studies for the treatment of febrile neutropenia and in the pediatric population.

Ceftobiprole medocaril is a water-soluble prodrug of ceftobiprole. This pyrrolidinone-3-ylidemethyl fifth-generation cephalosporin has activity against major gram-negative and gram-positive bacteria, including methicillin-resistant Staphylococcus aureus (MRSA) and ampicillin-sensitive Enterococcus spp. (Table 1) [76]. It is rapidly bactericidal against MRSA through inhibition of penicillin binding protein-2 and is effective against resistant strains with reduced susceptibility to vancomycin or expression of the Enterococcal vancomycin resistance gene complex [77]. Ceftobiprole has excellent bactericidal activity against Streptococcus spp., including those that are penicillin and macrolide resistant, as well as Haemophilus influenzae, Neisseria gonorrhoeae, and Moraxella catarrhalis [78-81]. Even though it does not have good activity against Acinetobacter baumanni complex (70.7\% resistant), it has more activity than ceftazidime and cefepime when $b l a_{A D C}$-like gene is repressed [82]. It has good concentrations in soft tissue, skeletal muscle, and adipose tissue and is effective in treating MRSA mediastinitis in rat models $[83,84]$. Ceftobiprole is studied at a dose of $500 \mathrm{mg} \mathrm{IV}$, infused over $2 \mathrm{~h}$ every $8 \mathrm{~h}$. It is $89 \%$ renally excreted and therefore requires dose reduction for renal impairment.

Ceftobiprole is not currently FDA approved in the USA but is approved in several countries for the treatment of acute bacterial skin and skin structure infections (ABSSSI) (Table 2-ceftobiprole medocaril). Initial RCTs investigated ceftobiprole for use of ABSSSI and demonstrated noninferiority to vancomycin plus ceftazidime for complicated ABSSSI including MRSA infections [33, 85]. There are several double-blind RCTs that evaluate the efficacy of ceftobiprole for the treatment of pneumonia [31-33]. Nicholson et al. found that ceftobiprole was noninferior to ceftriaxone plus linezolid for treatment of community-acquired bacterial pneumonia (CABP) [32]. However, when both 
groups were transitioned to oral therapy, eradications were better for the group receiving cefuroxime than ceftobiprole. A subsequent double-blind phase III RCT by Awad et al. included 781 patients and demonstrated noninferiority to ceftazidime plus linezolid for treatment of HABP, but not VABP, with comparable outcomes for Enterobacteriaceae and P. aeruginosa [31]. Notably, there were fewer patients in the VABP subgroup (96/781) and more studies are needed to determine its role in VABP. Current phase III studies have been initiated to evaluate efficacy in the treatment of bacteremia due to S. aureus.

$\mathrm{C} / \mathrm{T}$ is a combination of an oxyimino-aminothiazolyl cephalosporin with a $\beta$ lactamase inhibitor that has enhanced activity against class $A$ and $C \beta$ lactamases and some MBL (Table 1). C/T is FDA approved to be dosed at $1.5 \mathrm{~g}$ IV every $8 \mathrm{~h}$ for cUTI and CIAI and at a higher dose of $3 \mathrm{~g}$ IV every $8 \mathrm{~h}$ for nosocomial pneumonia [86]. C/T is more rapidly bactericidal than other cephalosporins [87]. It requires renal dose adjustment and $50-70 \%$ is removed with 3-4 h of hemodialysis [88]. Clearance is not significantly affected by weight, age, sex, ethnicity, or presence of infection [86]. Monogue et al. showed that patients with cystic fibrosis should receive over $90 \%$ probability of target attainment for $1.5 \mathrm{mg}$ every $8 \mathrm{~h}$ and 3 g every $8 \mathrm{~h}$ for $P$. aeruginosa MIC $\leq 4$ and 8 , respectively [89].

C/T was FDA approved in 2015 for the treatment of cUTI and in combination

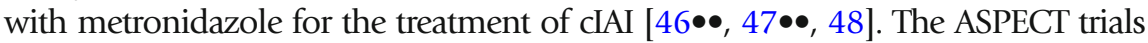
demonstrated that $\mathrm{C} / \mathrm{T}$ in combination with metronidazole was noninferior to meropenem for clinical cure of cIAI in 806 patients and superior to levofloxacin for composite and microbiological cures of cUTI in 1068 patients

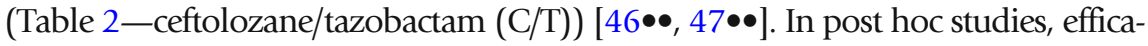
cy was maintained in patients with ESBL-producing isolates $(n=150), P$. aeruginos $(n=72)$, and in levofloxacin-resistant isolates $[43 \bullet, 44,45]$. Popejoy et al. showed that patients with diabetes mellitus in both treatment groups had lower cure rates and more adverse effects [39]. Moderate renal insufficiency was associated with higher rates of treatment failure for CIAI and cUTI regardless of treatment type [41]. Further investigation is necessary to determine the efficacy of treatment in these populations. Clinical trials are ongoing for the treatment of febrile infections due to MDR $P$. aeruginosa and VABP.

In observational studies, $\mathrm{C} / \mathrm{T}$ demonstrates efficacy with over $70-80 \%$ clinical success in the treatment of infections due to MDR and XDR P. aeruginosa, including those expressing amp-C and OXA-48 [35, 38, 40, 42, 90-92]. These studies consisted of patients with predominantly respiratory tract infections and included critically ill and immunocompromised hosts. Case reports suggest efficacy as rescue treatment for meningitis, osteomyelitis, and hardwareassociated infections due to XDR P. aeruginosa [34, 36, 37]. In vitro, the clinical success of C/T against MDR P. aeruginosa is attributed to its slow development of resistance, which requires multiple mutations in addition to amp-C production [93].

Delafloxacin is an anionic fluoroquinolone with increased activity against MRSA and gram-positive isolates (Table 1). It has a different structure than 
other quinolones that confers weak acidity, which allows it to be more active against $S$. aureus with improved intracellular and biofilm penetration [94]. Potency may be enhanced in the setting of a urinary tract infection in which urine is more acidic [95]. It has activity against gram-positive, anaerobe, and gram-negative organisms with better efficacy in vitro than levofloxacin for ciprofloxacin-resistant $S$. pneumoniae $[96,97]$. It is predominantly renally excreted with $58.8 \%$ bioavailability. The approved dose is $300 \mathrm{mg}$ every $12 \mathrm{~h} \mathrm{IV}$ or $450 \mathrm{mg}$ every $12 \mathrm{~h}$ orally $[98,99]$. Unlike other fluoroquinolones, it does not induce cytochrome p3A, does not prolong the QTc, and does not require dose reduction for hepatic dysfunction [98, 100-102].

Delafloxacin was first investigated and approved for the treatment of ABSSSI in 2017. In a phase III double-blind RCT of 660 patients, Pullman et al. demonstrated noninferiority to vancomycin plus aztreonam for objective clinical response at $48 \mathrm{~h}$ and microbiological eradication, which persisted in the MRSA subgroup analysis (Table 2-delafloxacin) [49••]. Phase II studies demonstrated comparable efficacy to tigecycline and linezolid and higher cure rates compared to vancomycin alone, particularly in obese patients $[50 \bullet$, 51]. Phase III studies were recently completed to evaluate the efficacy of delafloxacin for community-acquired pneumonia and uncomplicated urogenital gonorrhea with results pending.

\section{Eravacycline}

Eravacycline is a novel synthetic fluorocycline tetracycline with enhanced activity against gram-positive and gram-negative organisms, including anaerobes, and without activity against Pseudomonas spp. (Table 1) [103]. In vitro, it has activity for ESBL, KPC, and MBL such as OXA and NDM [104, 105]. It is structurally similar to tigecycline and evades many resistance mechanisms of other tetracyclines, including ribosomal protection proteins and efflux pumps [106]. Eravacycline was studied at a dose of $1 \mathrm{mg} / \mathrm{kg}$ every $12 \mathrm{~h} \mathrm{IV}$. It has linear pharmacokinetics with $28 \%$ oral bioavailability. In phase I and II studies, the most common adverse effects were dose-related nausea and superficial phlebitis in $87.5 \%$ [107]. Phase I studies indicate that eravacycline can maintain high concentrations in epithelial lining fluid and alveolar macrophages, supporting its potential role in treating respiratory infections [108].

Eravacycline was FDA approved for CIAI in 2018. The IGNITE 1 trial by Solomkin et al. demonstrated noninferiority to ertapenem for clinical cure of

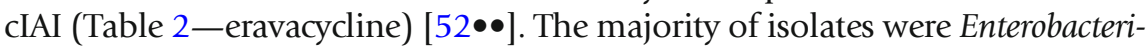

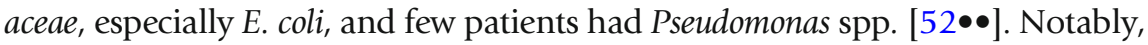
patients with renal insufficiency and hepatic dysfunction were excluded from this study. Prior phase II studies showed favorable response rates at both 1 and $1.5 \mathrm{mg} / \mathrm{kg}$ dosing compared to ertapenem [53]. A phase III trial for the treatment of cIAI compared to meropenem was completed with results pending. Two phase III trials for the treatment of cUTI were completed and failed to demonstrate noninferiority with results not currently published [109]. Phase I RCTs are recruiting patients to evaluate PK and safety in the pediatric population.

\section{Imipenem/cilastatin + relebactam}

Relebactam (MK7655) is a novel piperidine analogue diazabicyclooctane class $A$ and $C \beta$-lactamase inhibitor (Table 1). It is currently being studied in 
combination with imipenem and cilastatin (IMI) in the treatment of gramnegative infections due to $P$. aeruginosa, E. coli, K. pneumoniae, and Enterobacter spp. In vitro, the addition of relebactam reduces MICs for KPC K. pneumoniae by 64-fold, but it has less impact on other ESBL and amp-C producers and is not active against class $D \beta$-lactamases [110, 111]. It is predominantly renally excreted and phase I studies show that coadministration does not significantly affect PK of the individual agents [54•].

A phase II multicenter double-blind RCT by Lucasti et al. demonstrated noninferiority of relebactam at both 250 and $125 \mathrm{mg}$ doses in combination with IMI compared to IMI alone at discontinuation of IV therapy for treatment of cIAI (Table 2-imipenem/cilastatin (IMI) + relebactam (REL)) [54•]. However, only 36 isolates were IMI resistant [54•]. A phase III double-blind RCT comparing IMI/relebactam to IMI plus colistimethate for the treatment of IMIresistant bacterial infections completed recruitment in 2017 with results pending. A phase II study evaluating efficacy for cUTI was initiated as well. Clinical trials are recruiting to study the efficacy of IMI/relebactam versus piperacillin/ tazobactam for HABP/VABP, and phase I studies are underway in the pediatric population.

Lefamulin is a semisynthetic pleuromutilin that has a similar mechanism of action to oxazolidinones by interfering with protein synthesis through binding to the 50S ribosome (Table 1) [112]. Lefamulin has rapid and high tissue penetration in the epithelial lining of the lungs, which suggests its potential role in the treatment of respiratory tract infections [113]. It also has rapid tissue penetration into plasma, skeletal muscle, and subcutaneous adipose, suggesting a potential role for the treatment of ABSSSI [113]. It is active against all aerobic gram-positive organisms except Enterococcus faecalis, including activity against MRSA, vancomycin-intermediate $S$. aureus (VISA), vancomycin-resistant $S$. aureus (VRSA), penicillin-resistant $S$. pneumoniae, and vancomycin-resistant E. faecium $[112,114]$. It also has activity against gram-negative organisms (except $P$. aeruginosa and A. baumannii), atypical organisms, some anaerobes, and drug-resistant Neisseria gonorrhoeae $[112,115]$.

Lefamulin is not currently FDA approved but is being studied for the treatment of CABP and ABSSSI. A phase II RCT by Prince et al. with 210 patients demonstrated comparable clinical success in the treatment of ABSSSI with $100 \mathrm{mg}$ lefamulin, $150 \mathrm{mg}$ lefamulin, and vancomycin (Table 2-lefamulin (BC-3781)) [116]. Two phase III double-blind RCTs (LEAP 1 and 2) demonstrated lefamulin to be noninferior to moxifloxacin with or without linezolid for treatment of CABP, but full results and publications are pending $[55,117]$.

Vaborbactam (RPX7009) is a cyclic boronic acid $\beta$-lactamase inhibitor that inhibits class $\mathrm{A}$ and $\mathrm{C}$ as well as some class $\mathrm{D} \beta$-lactamases, that is currently being studied in combination with meropenem (Table 1). The coadministration of these agents does not alter their individual PK [118]. It is predominantly renally excreted with over $80 \%$ recovered in the urine [119].

Meropenem/vaborbactam was FDA approved for the treatment of cUTI based on the results of the TANGO I and II trials. The TANGO I trial by Kaye 
et al. included 545 patients and demonstrated noninferiority and superiority of meropenem/vaborbactam to piperacillin/tazobactam for clinical and microbiologic cure of cUTI, although only a limited number of the isolates were

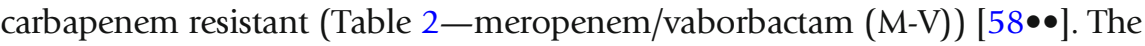
TANGO II trial by Wunderink et al. was a small study of 47 patients with CRE infections that demonstrated higher clinical and microbiological cure rates when treated with $\mathrm{M}-\mathrm{V}$ compared to the best available therapy (polymixins,

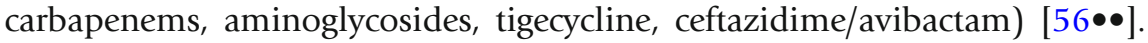
This study included 22 patients with BSI, and infections mostly due to K. pneumoniae, including MBL-producing organisms. Cure rates remained higher in immunocompromised patients and those with prior antibiotic failure

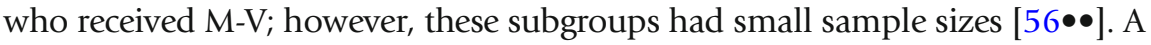
case report documents clinical and microbiologic success using $\mathrm{M}-\mathrm{V} 40 \mathrm{mg} / \mathrm{kg}$ IV every $6 \mathrm{~h}$ over $3 \mathrm{~h}$ to treat KPC K. pneumoniae bacteremia in a pediatric patient with successful target attainment [57].

\section{Plazomicin}

Plazomicin (ACHN-490) is an aminoglycoside derivative of sisomicin that inhibits protein synthesis by binding to the ribosomal 30 S subunit (Table 1). In phase I PK studies, it has predictable high peak and low troughs at $15 \mathrm{mg} / \mathrm{kg}$ once daily dosing, with achievable targets for E. coli and K. pneumoniae at $7 \mathrm{mg} / \mathrm{kg}$ [120]. Plazomicin has activity against typical aminoglycosideresistant pathogens, including those with aminoglycoside-modifying enzymes (AME), ESBL, KPC, and MBL. In vitro, plazomicin has good activity against Enterobacteriaceae, including CRE and KPC, which is comparable to amikacin, carbapenems, and tigecycline, and better than gentamycin and tobramycin [121, 122]. In vitro, it inhibits $84.6 \% \mathrm{CRE}, 92.9 \% \mathrm{KPC}$ without MBL, and $87 \%$ OXA-48 and has improved activity against isolates with AME encoding genes (99\% inhibited overall) compared to other aminoglycosides (gentamycin $31.3 \%$, tobramycin $1.5 \%$, amikacin $76.9 \%)$, meropenem $(78.2 \%)$, and tigecycline (94.6\%) [122]. Plazomicin has activity against Acinetobacter baumannii, Acinetobacter calcoaceticus, and P. aeruginosa; however, break points are higher for these organisms. It has limited activity against Enterococcus spp., S. pneumoniae, Providencia spp., Morganella spp., and Proteus mirabilis [121]. In vitro, it is the only aminoglycoside active against Brucella suis, but it is not consistently active against other Brucella spp. [123].

Plazomicin is currently FDA approved for treatment of cUTI. A phase II double-blind RCT by Connolly et al. demonstrated comparable clinical and microbiological cure for $10 \mathrm{mg} / \mathrm{kg}$ plazomicin, $15 \mathrm{mg} / \mathrm{kg}$ plazomicin, and levofloxacin for the treatment of cUTI and acute pyelonephritis (Table 2-plazomicin) [59•]. In this study, 2 of 74 patients at $15 \mathrm{mg} / \mathrm{kg}$ had vestibular or cochlear-related adverse effects and two patients discontinued therapy due to nephrotoxicity [59•]. Phase III RCTs that compare efficacy to meropenem for the treatment of cUTI and to colistin for CRE infections completed recruitment with results pending.

\section{Solithromycin}

Solithromycin is a fourth-generation macrolide fluoroketolide (Table 1). It has $67 \%$ bioavailability and is not affected by food. It is metabolized by 
cyp3A4p450 and is predominantly biliary excreted with only $10 \%$ renal clearance, but does not require dose adjustment for hepatic impairment [124]. It does not affect QTc and has similar safety for adolescents as adults [125, 126]. It has high concentrations in epithelial lining fluid and alveolar macrophages, suggesting a potential role in treating respiratory tract infections [127]. Its unique chemical structure allows greater intracellular accumulation than other macrolides, enabling it to treat both extra- and intracellular pathogens. Solithromycin has activity in vitro against both gram-positive and gramnegative organisms, including gram-positive anaerobes [128]. It has increased activity for Mycoplasma genitalium in vitro compared to other macrolides and 8to 32-fold higher activity for Legionella pneumophila serotype 1 than azithromycin [129, 130].

Solithromycin is not currently FDA approved but is being studied for the treatment of CABP and urogenital gonorrhea. The SOLITAIRE-oral and SOLITAIRE-IV studies demonstrated that 5 days of treatment with solithromycin was noninferior to moxifloxacin for the treatment of CABP based on early clinical response, and clinical cure at short- and long-term follow-up


$800 \mathrm{mg}$ orally or IV, followed by $400 \mathrm{mg}$ PO daily for days 2 through 5 [60••, $61 \bullet \bullet$. Initial phase II studies compared solithromycin to levofloxacin with comparable efficacy [131]. Phase II studies were initiated to evaluate efficacy of a single $1200 \mathrm{mg}$ PO dose for the treatment of N. gonorrhea [63]. A phase III study (SOLITAIR-J) to evaluate efficacy and safety of a single dose of $1200 \mathrm{mg}$ PO solithromycin compared to intramuscular ceftriaxone and azithromycin for the treatment of uncomplicated urogenital gonorrhea with or without concomitant Chlamydia trachomatis completed enrollment with results pending.

Solupenem is a carbapenem that is available orally and IV. It is active against gram-positive organisms and gram-negative organisms, including ESBL, but is not active against $P$. aeruginosa, Enterococcus spp., CRE, or MRSA (Table 1) [62]. It is dosed either at $1000 \mathrm{mg}$ IV once daily or $500 \mathrm{mg}$ PO twice daily with probenecid $500 \mathrm{mg}$ PO twice daily.

Sulopenem is not FDA approved but being studied for CABP and cIAI. A phase II double-blind RCT was conducted to evaluate its efficacy for CABP requiring hospitalization with switch to oral therapy compared to ceftriaxone with transition to amoxicillin/clavulanic acid, with results pending. A phase III RCT is recruiting patients to compare solupenem to ertapenem for the treatment of cIAI.

Given the antimicrobial resistance crisis, the development of new antimicrobials with enhanced activity against drug-resistant bacteria is critical; this is especially true for resistant gram-negative organisms. Of the agents that are currently in the pipeline or recently approved, most represent modifications of preexisting agents and the majority are administered intravenously. Each agent, except cefiderocol, targets only certain classes of $\beta$-lactamases and there continues to be a lack of treatment options for infections caused by metallo- $\beta$ - 
lactamase-producing organisms. The use of ceftazidime/avibactam and ceftolozane/tazobactam for the treatment of resistant gram-negative infections is promising but studies looking specifically at patients with infections due to MDR organisms remain sparse and are limited to observational studies. The majority of RCTs for new antimicrobials have focused on cUTI and CIAI, and their effectiveness in other severe infections including BSI and nosocomial pneumonia is unclear. More studies are needed to determine the role of these agents in the treatment of resistant gram-negative infections.

\section{Compliance with Ethical Standards}

\section{Conflict of Interest}

Nicole C. Vissichelli declares that she has no conflict of interest.

Michael P. Stevens declares that he has no conflict of interest.

Human and Animal Rights and Informed Consent

The article does not contain any studies with human or animal subjects performed by any of the authors.

\section{References and Recommended Readings}

Papers of particular interest, published recently, have been highlighted as:

- Of importance

$\bullet \quad$ Of major importance

1. Talbot GH, Jezek A, Murray BE, Jones RN, Ebright RH, Nau GJ, et al. The Infectious Diseases Society of America's $10 \times$ '20 initiative (ten new systemic antibacterial agents FDA-approved by 2020): Is $20 \times$ '20 a possibility? Clin Infect Dis. 2019. [Epub ahead of print].

2. Antimicrobial resistance global report on surveillance. World Health Organization. 2014. [Internet] [cited 2019 02 18]. Available from: https://apps.who.int/iris/bitstream/ handle/10665/112642/9789241564748_eng.pdf;jsessionid= 33FAEEDFE1183392D13CD4E1139521BC?sequence=1

3. U.S. challenges world to intensify global fight against antibiotic resistance | CDC Online Newsroom | CDC [Internet]. [cited 2019 Feb 18]. Available from: https:// www.cdc.gov/media/releases/2018/p0925-globalantibiotic-resistance.html

4. Saisho Y, Katsube T, White S, Fukase H, Shimada J. Pharmacokinetics, safety, and tolerability of cefiderocol, a novel siderophore cephalosporin for gram-negative bacteria, in healthy subjects. Antimicrob Agents Chemother. American Society for Microbiology Journals. 2018;62:e02163-17.

5. Katsube T, Miyazaki S, Narukawa Y, Hernandez-Illas M, Wajima T. Drug-drug interaction of cefiderocol, a siderophore cephalosporin, via human drug transporters. Eur J Clin Pharmacol.

Springer, Berlin. 2018;74:931-8.

6. Ghazi IM, Monogue ML, Tsuji M, Nicolau DP. Humanized exposures of cefiderocol, a siderophore cephalosporin, display sustained in vivo activity against siderophore-resistant Pseudomonas aeruginosa. Pharmacology.

Karger Publishers. 2018;101:278-84.

7. Ito A, Kohira N, Bouchillon SK, West J, Rittenhouse S, Sader HS, et al. In vitro antimicrobial activity of S-649266, a catechol-substituted siderophore cephalosporin, when tested against non-fermenting Gram-negative bacteria. J Antimicrob Chemother. 2016;71:670-7.

8. Kohira N, West J, Ito A, Ito-Horiyama T, Nakamura R, Sato $\mathrm{T}$, et al. In vitro antimicrobial activity of a siderophore cephalosporin, S-649266, against Enterobacteriaceae clinical isolates, including carbapenemresistant strains. Antimicrob Agents Chemother.

American Society for Microbiology Journals. 2016;60:729-34.

9.• Portsmouth S, van Veenhuyzen D, Echols R, Machida M, Ferreira JCA, Ariyasu M, et al. Cefiderocol versus imipenem-cilastatin for the treatment of complicated urinary tract infections caused by Gram-negative uropathogens: a phase 2, randomised, double-blind, non-inferiority trial. Lancet Infect Dis. 2018;18:1319-28. This phase II study determined cefiderocol to be non-inferior to imipenem/cilastatin for the treatment of complicated urinary tract infections and acute pyelonephritis. 
10. De la Calle C, Rodríguez O, Morata L, Marco F, Cardozo C, García-Vidal C, et al. Clinical characteristics and prognosis of infections caused by OXA-48 carbapenemase producing Enterobacteriaceae in patients treated with ceftazidime-avibactam. Int J Antimicrob Agents. 2019;53(4):520-524.

11. Gofman N, To K, Whitman M, Garcia-Morales E. Successful treatment of ventriculitis caused by Pseudomonas aeruginosa and carbapenem-resistant Klebsiella pneumoniae with i.v. ceftazidime-avibactam and intrathecal amikacin. Am J Health Syst Pharm. 2018;75:953-7.

12. Sousa A, Pérez-Rodríguez MT, Soto A, Rodríguez L, Pérez-Landeiro A, Martínez-Lamas L, et al. Effectiveness of ceftazidime/avibactam as salvage therapy for treatment of infections due to OXA-48 carbapenemaseproducing Enterobacteriaceae. J Antimicrob Chemother. 2018;73:3170-5.

This article is a prospective observational study that demonstrates $77 \%$ clinical success utilizing ceftazidime/avibactam for the treament of infections due to OXA-48 Carbapenemase producing Enterobacteriaceae. It is the only current prospective study that focuses on the utility of ceftazidime-avibactam in infections due to OXA-48 producing CRE.

13. Rodríguez-Núñez O, Ripa M, Morata L, de la Calle C, Cardozo C, Fehér C, et al. Evaluation of ceftazidime/ avibactam for serious infections due to multidrugresistant and extensively drug-resistant Pseudomonas aeruginosa. J Glob Antimicrob Resist. 2018;15:136-9.

14. Mittal J, Szymczak WA, Guo Y, Levi MH, Chen L, Kreiswirth BN, et al. Two for the price of one: emerging carbapenemases in a returning traveller to New York City. BMJ Case Rep.

BMJ Publishing Group Ltd. 2018;2018:bcr-2018-225440.

15. Santevecchi BA, Smith TT, MacVane SH. Clinical experience with ceftazidime/avibactam for treatment of antibiotic-resistant organisms other than Klebsiella pneumoniae. Int J Antimicrob Agents. 2018;51:629-35.

16.• Torres A, Zhong N, Pachl J, Timsit J-F, Kollef M, Chen Z, et al. Ceftazidime-avibactam versus meropenem in nosocomial pneumonia, including ventilatorassociated pneumonia (REPROVE): a randomised, double-blind, phase 3 non-inferiority trial. Lancet Infect Dis. 2018;18:285-95.

This phase III clinical trial demonstrates that ceftazidimeavibactam was non-inferior to meropenem in the treatment of nosocomial pneumonia, prompting FDA approval for this indication.

17. Van Duin D, Lok JJ, Earley M, Cober E, Richter SS, Perez $\mathrm{F}$, et al. Colistin versus ceftazidime-avibactam in the treatment of infections due to carbapenem-resistant Enterobacteriaceae. Clin Infect Dis AColistin vs Ceftazidime-Avibactam CRE Infect • CID. 2018;66:163.

This is the first prospective study to investigate the utility of ceftazidime/avibactam specifically for KPC producing CRE infections. Those treated with ceftazidime-avibactam had better outcomes than those treated with colistin with a significantly lower all-cause mortality.
18. Mariano N, Philp T, Bulbin A, Bono C, Urban C. Successful treatment of Klebsiella pneumoniae harboring a Klebsiella pneumoniae carbapenemase isolated from lumbar wound infection and blood in a patient with hardware retention. Case Rep Infect Dis.

Hindawi Limited. 2017;2017:1-5.

19. King M, Heil E, Kuriakose S, Bias T, Huang V, El-Beyrouty $\mathrm{C}$, et al. Multicenter study of outcomes with ceftazidimeavibactam in patients with carbapenem-resistant Enterobacteriaceae infections. Antimicrob Agents Chemother.

American Society for Microbiology Journals. 2017;61:e0044917.

20. Castón JJ, Lacort-Peralta I, Martín-Dávila P, Loeches B, Tabares S, Temkin L, et al. Clinical efficacy of ceftazidime/avibactam versus other active agents for the treatment of bacteremia due to carbapenemaseproducing Enterobacteriaceae in hematologic patients. Int J Infect Dis. 2017;59:118-23.

21. Krapp F, Grant JL, Sutton SH, Ozer EA, Barr VO. Treating complicated carbapenem-resistant enterobacteriaceae infections with ceftazidime/avibactam: a retrospective study with molecular strain characterisation. Int J Antimicrob Agents.

Elsevier. 2017;49:770-3.

22.• Qin X, Tran BG, Kim MJ, Wang L, Nguyen DA, Chen Q, et al. A randomised, double-blind, phase 3 study comparing the efficacy and safety of ceftazidime/ avibactam plus metronidazole versus meropenem for complicated intra-abdominal infections in hospitalised adults in Asia. Int J Antimicrob Agents. Elsevier. 2017;49:579-88.

This phase III clinical trial demonstrates non-inferiority of ceftazidime/avibactam to meropenem when used in combination with metronidazole for the treatment of complicated intra-abdominal infections in Asia.

23. Shields RK, Nguyen MH, Chen L, Press EG, Potoski BA, Marini RV, et al. Ceftazidime-avibactam is superior to other treatment regimens against carbapenem-resistant Klebsiella pneumoniae bacteremia. Antimicrob Agents Chemother. 2017;61.

24. Mendes RE, Castanheira M, Woosley LN, Stone GG, Bradford PA, Flamm RK. Molecular $\beta$-lactamase characterization of aerobic gram-negative pathogens recovered from patients enrolled in the ceftazidimeavibactam phase 3 trials for complicated intraabdominal infections, with efficacies analyzed against susceptible and resistant subsets. Antimicrob Agents Chemother. American Society for Microbiology Journals. 2017;61:e02447-16.

This study demonstrates similar cure rates for patients treated with ceftazidime avibactam plus metronidazole in patients enrolled in the phase III clinical trials with intraabdominal infections due to ESBL or carbapenemase producing organisms.

25. Shields RK, Chen L, Cheng S, Chavda KD, Press EG, Snyder A, et al. Emergence of ceftazidime-avibactam resistance due to plasmid-borne blaKPC-3 mutations during treatment of carbapenem-resistant Klebsiella pneumoniae infections. Antimicrob Agents Chemother. 
American Society for Microbiology Journals. 2017;61:e0209716.

26. Shields RK, Potoski BA, Haidar G, Hao B, Doi Y, Chen L, et al. Clinical outcomes, drug toxicity, and emergence of ceftazidime-avibactam resistance among patients treated for carbapenem-resistant Enterobacteriaceae infections: Table 1. Clin Infect Dis. 2016;63:1615-8.

27. Carmely, PY, Armstrong J, Laud PJ, Newell P, Stone G, Wardman A, Gasink LB. Ceftazidimeavibactam or best available therapy in patients with ceftazidime-resistant Enterobacteriaceae and Pseudomonas aeruginosa complicated urinary tract infections or complicated intraabdominal infections (REPRISE): a randomized, pathogen-directed, phase 3 study. Lancet Infectious Diseases. 2016;16:661-73.

This phase III trial demonstrated the efficacy of ceftazidimeavibactam compared to bestavailable therapy for the treatment of complicated urinary tract infections or complicated intraabdominal infections due to ceftazidime-resistant isolates.

28.• Wagenlehner FM, Sobel JD, Newell P, Armstrong J, Huang X, Stone GG, et al. Ceftazidime-avibactam versus doripenem for the treatment of complicated urinary tract infections, including acute pyelonephritis: RECAPTURE, a phase 3 randomized trial program. Clin Infect Dis. Oxford University Press. 2016;63:754-62. This phase III clinical trial demonstrates non-inferiority of ceftazidime-avibactam to doripenem for the treatment of complicated urinary tract infections, prompting FDA approval for this indication.

29.• Mazuski JE, Gasink LB, Armstrong J, Broadhurst H, Stone GG, Rank D, et al. Efficacy and safety of ceftazidime-avibactam plus metronidazole versus meropenem in the treatment of complicated intraabdominal infection: results from a randomized, controlled, double-blind, phase 3 program. Clin Infect Dis. Oxford University Press. 2016;62:1380-9.

This phase III clinical trial prompted FDA approval of ceftazidime-avibactam for the treatment of complicated intraadominal infections in combination with metronidazole with non-inferiority to meropenem.

30. Mendes RE, Castanheira M, Gasink L, Stone GG, Nichols WW, Flamm RK, et al. $\beta$-Lactamase characterization of gram-negative pathogens recovered from patients enrolled in the phase 2 trials for ceftazidimeavibactam: clinical efficacies analyzed against subsets of molecularly characterized isolates. Antimicrob Agents Chemother.

American Society for Microbiology Journals. 2015;60:132835.

31. Awad SS, Rodriguez AH, Chuang Y-C, Marjanek Z, Pareigis AJ, Reis G, et al. A phase 3 randomized doubleblind comparison of ceftobiprole medocaril versus ceftazidime plus linezolid for the treatment of hospital-acquired pneumonia. Clin Infect Dis.

Oxford University Press. 2014;59:51-61.

32. Nicholson SC, Welte T, File TM, Strauss RS, Michiels B, Kaul $P$, et al. A randomised, double-blind trial comparing ceftobiprole medocaril with ceftriaxone with or without linezolid for the treatment of patients with community-acquired pneumonia requiring hospitalisation. Int J Antimicrob Agents.

Elsevier. 2012;39:240-6.

33. Noel GJ, Bush K, Bagchi P, Ianus J, Strauss RS. A randomized, double-blind trial comparing ceftobiprole medocaril with vancomycin plus ceftazidime for the treatment of patients with complicated skin and skinstructure infections. Clin Infect Dis.

Oxford University Press. 2008;46:647-55.

34. Frattari A, Savini V, Polilli E, Cibelli D, Talamazzi S, Bosco D, et al. Ceftolozane-tazobactam and fosfomycin for rescue treatment of otogenous meningitis caused by XDR Pseudomonas aeruginosa: case report and review of the literature. IDCases. 2018;14:e00451.

35. Bassetti M, Castaldo N, Cattelan A, Mussini C, Righi E, Tascini C, et al. Ceftolozane/tazobactam for the treatment of serious Pseudomonas aeruginosa infections: a multicentre nationwide clinical experience. Int J Antimicrob Agents. 2018.

36. Hassan S, Kahn MD, Saraiya N, Nori P. Treatment of a complex orthopaedic infection due to extensively drugresistant Pseudomonas aeruginosa. BMJ Case Rep.

BMJ Publishing Group. 2018;2018:bcr-2017-223202.

37. Dietl B, Sánchez I, Arcenillas P, Cuchi E, Gómez L, González de Molina FJ, et al. Ceftolozane/tazobactam in the treatment of osteomyelitis and skin and softtissue infections due to extensively drug-resistant Pseudomonas aeruginosa: clinical and microbiological outcomes. Int J Antimicrob Agents. 2018;51:498-502.

38. Haidar G, Philips NJ, Shields RK, Snyder D, Cheng S, Potoski BA, et al. Ceftolozane-tazobactam for the treatment of multidrug-resistant Pseudomonas aeruginosa infections: clinical effectiveness and evolution of resistance. Clin Infect Dis.

Oxford University Press. 2017;65:110-20.

39. Popejoy MW, Long J, Huntington JA. Analysis of patients with diabetes and complicated intra-abdominal infection or complicated urinary tract infection in phase 3 trials of ceftolozane/tazobactam. BMC Infect Dis.

BioMed Central. 2017;17:316.

40. Munita JM, Aitken SL, Miller WR, Perez F, Rosa R, Shimose LA, et al. Multicenter evaluation of ceftolozane/tazobactam for serious infections caused by carbapenem-resistant Pseudomonas aeruginosa. Clin Infect Dis.

Oxford University Press. 2017;65:158-61.

41. Kullar R, Wagenlehner FM, Popejoy MW, Long J, Yu B, Goldstein EJC. Does moderate renal impairment affect clinical outcomes in complicated intra-abdominal and complicated urinary tract infections? Analysis of two randomized controlled trials with ceftolozane/tazobactam. J Antimicrob Chemother.

Oxford University Press. 2016;72:dkw486.

42. Castón JJ, De la Torre Á, Ruiz-Camps I, Sorlí ML, Torres $\mathrm{V}$, Torre-Cisneros J. Salvage therapy with ceftolozanetazobactam for multidrug-resistant Pseudomonas aeruginosa infections. Antimicrob Agents Chemother. 
American Society for Microbiology Journals. 2017;61:e0213616.

43. Popejoy MW, Paterson DL, Cloutier D, Huntington JA, Miller B, Bliss CA, et al. Efficacy of ceftolozane/ tazobactam against urinary tract and intra-abdominal infections caused by ESBL-producing Escherichia coli and Klebsiella pneumoniae: a pooled analysis of phase 3 clinical trials. J Antimicrob Chemother. Oxford University Press. 2017;72:268-72.

This study demonstrated efficacy in treating complicate intraabdominal and urinary tract infections due to ESBL Enterobacteriaceae with ceftolozane/tazobactam.

44. Miller B, Popejoy MW, Hershberger E, Steenbergen JN, Alverdy J. Characteristics and outcomes of complicated intra-abdominal infections involving Pseudomonas aeruginosa from a randomized, double-blind, phase 3 ceftolozane-tazobactam study. Antimicrob Agents Chemother.

American Society for Microbiology Journals. 2016;60:438790.

45. Huntington JA, Sakoulas G, Umeh O, Cloutier DJ, Steenbergen JN, Bliss C, et al. Efficacy of ceftolozane/ tazobactam versus levofloxacin in the treatment of complicated urinary tract infections (cUTIs) caused by levofloxacin-resistant pathogens: results from the ASPECT-cUTI trial. J Antimicrob Chemother. 2016;71:2014-21.

46.• Wagenlehner FM, Umeh O, Steenbergen J, Yuan G, Darouiche RO. Ceftolozane-tazobactam compared with levofloxacin in the treatment of complicated urinary-tract infections, including pyelonephritis: a randomised, double-blind, phase 3 trial (ASPECT-cUTI). Lancet. Elsevier. 2015;385:1949-56.

This phase III study demonstrated non-inferiority and superiority of ceftolozane/tazobactam to levofloxacin for the treatment of complicated urinary tract infections. This prompted FDA approval of ceftolozane/tazobactam for this indication.

47.• Solomkin J, Hershberger E, Miller B, Popejoy M, Friedland I, Steenbergen J, et al. Ceftolozane/ tazobactam plus metronidazole for complicated intraabdominal infections in an era of multidrug resistance: results from a randomized, double-blind, phase 3 trial (ASPECT-cIAI). Clin Infect Dis. Oxford University Press. 2015;60:1462-71.

This phase III study demonstrated non-inferiority of ceftolozane/tazobactam in combination with metronidazole to meropenem for the treatment of complicated intraabdominal infections. This prompted FDA approval of ceftolozane/tazobactam for this indication.

48. Lucasti C, Hershberger E, Miller B, Yankelev S, Steenbergen J, Friedland I, et al. Multicenter, doubleblind, randomized, phase II trial to assess the safety and efficacy of ceftolozane-tazobactam plus metronidazole compared with meropenem in adult patients with complicated intra-abdominal infections. Antimicrob Agents Chemother.

American Society for Microbiology Journals. 2014;58:5350-7. 49.•P Pullman J, Gardovskis J, Farley B, Sun E, Quintas M, Lawrence $\mathrm{L}$, et al. Efficacy and safety of delafloxacin compared with vancomycin plus aztreonam for acute bacterial skin and skin structure infections: a phase 3, double-blind, randomized study. J Antimicrob Chemother. Oxford University Press. 2017;72:347180.

This phase III clinical trial demonstrates that delafloxacin is non-inferior to vancomycin plus aztreonam for the treatment of acute bacterial skin and soft tissue infections, prompting FDA approval for this indication.

50. Kingsley J, Mehra P, Lawrence LE, Henry E, Duffy E, Cammarata SK, et al. A randomized, double-blind, phase 2 study to evaluate subjective and objective outcomes in patients with acute bacterial skin and skin structure infections treated with delafloxacin, linezolid or vancomycin. J Antimicrob Chemother. Oxford University Press. 2016;71:821-9.

This study demonstrated that delafloxacin had comparable efficacy to tigecycline and linezolid and higher cure rates compared to vancomycin alone for the treatment of skin and skin structure infections.

51. O'Riordan W, Mehra P, Manos P, Kingsley J, Lawrence $\mathrm{L}$, Cammarata S. A randomized phase 2 study comparing two doses of delafloxacin with tigecycline in adults with complicated skin and skin-structure infections. Int J Infect Dis.

Elsevier. 2015;30:67-73.

52.• Solomkin J, Evans D, Slepavicius A, Lee P, Marsh A, Tsai $\mathrm{L}$, et al. Assessing the efficacy and safety of eravacycline vs ertapenem in complicated intra-abdominal infections in the Investigating Gram-Negative Infections Treated with Eravacycline (IGNITE 1) trial. JAMA Surg. American Medical Association. 2017;152:224.

This phase III clinical trial demonstrated that eravacycline was non-inferior to erapenem for the treatment of complicated intraabdoiminal infections, prompting FDA approval for this indication.

53. Solomkin JS, Ramesh MK, Cesnauskas G, Novikovs N, Stefanova P, Sutcliffe JA, et al. Phase 2, randomized, double-blind study of the efficacy and safety of two dose regimens of eravacycline versus ertapenem for adult community-acquired complicated intraabdominal infections. Antimicrob Agents Chemother. American Society for Microbiology (ASM). 2014;58:1847-54.

54. Lucasti C, Vasile L, Sandesc D, Venskutonis D, McLeroth P, Lala M, et al. Phase 2, dose-ranging study of relebactam with imipenem-cilastatin in subjects with complicated intra-abdominal infection. Antimicrob Agents Chemother. American Society for Microbiology Journals. 2016;60:6234-43.

This phase II clinical trial demonstrates efficacy of using imipenem/cilistatin plus relebactam for the treatment of complicated intraabdominal infections, with noninferiority to imipenem alone.

55. File T, Goldberg L, Paukner S, Das A, Gelone SP, Saviski J, et al. 2386. Efficacy of lefamulin (LEF) vs moxifloxacin (MOX) against common pathogens in adults with community-acquired bacterial pneumonia (cabp): results from the phase 3 Lefamulin Evaluation 
Against Pneumonia (LEAP 1) study. Open Forum Infect Dis.

Oxford University Press. 2018;5:S711-2.

56.• Wunderink RG, Giamarellos-Bourboulis EJ, Rahav G, Mathers AJ, Bassetti M, Vazquez J, et al. Effect and safety of meropenem-vaborbactam versus best-available therapy in patients with carbapenem-resistant Enterobacteriaceae infections: the TANGO II randomized clinical trial. Infect Dis Ther. 2018.

This is the first phase III clinical trial to investigate efficacy of novel antibiotics specifically for CRE infections. Patients, especially those with prior antibiotic failure and immunocompromised hosts, who were treated with meropenemvaborbactam had improved outcomes compared to bestavailable therapy for CRE infections.

57. Hanretty AM, Kaur I, Evangelista AT, Ii WSM, Enache A Chopra A, et al. Pharmacokinetics of the meropenem component of meropenem-vaborbactam in the treatment of KPC-producing Klebsiella pneumoniae bloodstream infection in a pediatric patient. Pharmacotherapy. 2018;38(12):e87-91.

58.• Kaye KS, Bhowmick T, Metallidis S, Bleasdale SC, Sagan OS, Stus V, et al. Effect of meropenem-vaborbactam vs piperacillin-tazobactam on clinical cure or improvement and microbial eradication in complicated urinary tract infection. JAMA. American Medical Association. 2018;319:788

This phase III study demonstrates non-inferiority for meropenem-vaborbactam versus piperacillin-tazobactam for the treatment of complicated urinary tract infections, prompting FDA approval for this indication.

59. Connolly LE, Riddle V, Cebrik D, Armstrong ES, Miller LG. A multicenter, randomized, double-blind, phase 2 study of the efficacy and safety of plazomicin compared with levofloxacin in the treatment of complicated urinary tract infection and acute pyelonephritis. Antimicrob Agents Chemother. American Society for Microbiology Journals. 2018;62:e01989-17.

This phase II RCT demonstrates the efficacy of plazomicin for the treatment of complicated urinary tract infections.

60.• File TM, Rewerska B, Vucinić-Mihailović V, Gonong JRV, Das AF, Keedy K, et al. SOLITAIRE-IV: a randomized, double-blind, multicenter study comparing the efficacy and safety of intravenous-to-oral solithromycin to intravenous-to-oral moxifloxacin for treatment of community-acquired bacterial pneumonia. Clin Infect Dis. Oxford University Press. 2016;63:1007-16.

This phase III clinical trial demonstrates non-inferiority of solithromycin compared to moxifloxacin for the treatment of community acquired pneumonia using intial IV followed by oral therapy.

61.•• Barrera CM, Mykietiuk A, Metev H, Nitu MF, Karimjee N, Doreski PA, et al. Efficacy and safety of oral solithromycin versus oral moxifloxacin for treatment of community-acquired bacterial pneumonia: a global, double-blind, multicentre, randomised, active-controlled, non-inferiority trial (SOLITAIRE-ORAL). Lancet Infect Dis. Elsevier. 2016;16:421-30.

This phase III clinical trial demonstrated that oral solithromycin is non-inferior to moxifloxacin for the treatment of community acquired pneumonia.

62. Kosowska-Shick K, Ednie LM, McGhee P, Appelbaum PC. Comparative antipneumococcal activities of sulopenem and other drugs. Antimicrob Agents Chemother

American Society for Microbiology Journals. 2009;53:223947.

63. Hook EW, Golden M, Jamieson BD, Dixon PB, Harbison HS, Lowens S, et al. A phase 2 trial of oral solithromycin $1200 \mathrm{mg}$ or $1000 \mathrm{mg}$ as single-dose oral therapy for uncomplicated gonorrhea. Clin Infect Dis.

Oxford University Press. 2015;61:1043-8.

64. Mutters NT, Zimmermann S, Kaase M, Mischnik A. Activity of temocillin, mecillinam, ceftazidime, and ceftazidime/avibactam against carbapenem-nonsusceptible Enterobacteriaceae without carbapenemase production. Eur J Clin Microbiol Infect Dis. 2015;34:2429-37.

65. Stachyra T, Levasseur P, Péchereau M-C, Girard A-M, Claudon $\mathrm{M}$, Miossec $\mathrm{C}$, et al. In vitro activity of the \{beta\}-lactamase inhibitor NXL104 against KPC-2 carbapenemase and Enterobacteriaceae expressing KPC carbapenemases. J Antimicrob Chemother. 2009;64:326-9.

66. Gaibani P, Campoli C, Lewis RE, Volpe SL, Scaltriti E, Giannella $\mathrm{M}$, et al. In vivo evolution of resistant subpopulations of KPC-producing Klebsiella pneumoniae during ceftazidime/avibactam treatment. J Antimicrob Chemother.

Oxford University Press. 2018;73:1525-9.

67. Zhanel GG, Lawson CD, Adam H, Schweizer F, Zelenitsky S, Lagacé-Wiens PRS, et al. Ceftazidimeavibactam: a novel cephalosporin/ $\beta$-lactamase inhibitor combination. Drugs.

Springer International Publishing AG. 2013;73:159-77.

68. Tarral A, Merdjan H. Effect of age and sex on the pharmacokinetics and safety of avibactam in healthy volunteers. Clin Ther.

Elsevier. 2015;37:877-86.

69. Bradley JS, Armstrong J, Arrieta A, Bishai R, Das S, Delair S, et al. Phase I study assessing the pharmacokinetic profile, safety, and tolerability of a single dose of ceftazidime-avibactam in hospitalized pediatric patients. Antimicrob Agents Chemother.

American Society for Microbiology Journals. 2016;60:6252-9.

70. Bensman TJ, Wang J, Jayne J, Fukushima L, Rao AP, D'Argenio DZ, et al. Pharmacokinetic-

pharmacodynamic target attainment analyses to determine optimal dosing of ceftazidime-avibactam for the treatment of acute pulmonary exacerbations in patients with cystic fibrosis. Antimicrob Agents Chemother.

American Society for Microbiology Journals. 2017;61:e0098817.

71. Merdjan H, Tarral A, Das S, Li J. Phase 1 study assessing the pharmacokinetic profile and safety of avibactam in patients with renal impairment. J Clin Pharmacol. 2017;57:211-8. 
72. Das S, Li J, Armstrong J, Learoyd M, Edeki T. Randomized pharmacokinetic and drug-drug interaction studies of ceftazidime, avibactam, and metronidazole in healthy subjects. Pharmacol Res Perspect.

2015;3:e00172.

73. Nicolau DP, Siew L, Armstrong J, Li J, Edeki T, Learoyd $\mathrm{M}$, et al. Phase 1 study assessing the steady-state concentration of ceftazidime and avibactam in plasma and epithelial lining fluid following two dosing regimens. J Antimicrob Chemother. 2015;70:2862-9.

74. Lucasti C, Popescu I, Ramesh MK, Lipka J, Sable C. Comparative study of the efficacy and safety of ceftazidime/avibactam plus metronidazole versus meropenem in the treatment of complicated intraabdominal infections in hospitalized adults: results of a randomized, double-blind, phase II trial. J Antimicrob Chemother.

Oxford University Press. 2013;68:1183-92.

75. Stone GG, Newell P, Gasink LB, Broadhurst H, Wardman A, Yates K, et al. Clinical activity of ceftazidime/avibactam against MDR Enterobacteriaceae and Pseudomonas aeruginosa: pooled data from the ceftazidime/avibactam phase III clinical trial programme. J Antimicrob Chemother.

Oxford University Press. 2018;73:2519-23.

76. Zbinden R, Pünter V, von Graevenitz A. In vitro activities of BAL9141, a novel broad-spectrum pyrrolidinone cephalosporin, against gram-negative nonfermenters. Antimicrob Agents Chemother.

American Society for Microbiology Journals. 2002;46:871-4.

77. Chung M, Antignac A, Kim C, Tomasz A. Comparative study of the susceptibilities of major epidemic clones of methicillin-resistant Staphylococcus aureus to oxacillin and to the new broad-spectrum cephalosporin ceftobiprole. Antimicrob Agents Chemother.

American Society for Microbiology Journals. 2008;52:270917.

78. Betriu C, Culebras E, Gómez M, López-Fabal F, Rodríguez-Avial I, Picazo JJ. Comparative in vitro activity of ceftobiprole against Gram-positive cocci. Int J Antimicrob Agents.

Elsevier. 2010;36:111-3.

79. Kosowska K, Hoellman DB, Lin G, Clark C, Credito K, McGhee P, et al. Antipneumococcal activity of ceftobiprole, a novel broad-spectrum cephalosporin. Antimicrob Agents Chemother.

American Society for Microbiology Journals. 2005;49:193242.

80. Murthy B, Schmitt-Hoffmann A. Pharmacokinetics and pharmacodynamics of ceftobiprole, an anti-MRSA cephalosporin with broad-spectrum activity. Clin Pharmacokinet.

Springer International Publishing. 2008;47:21-33.

81. Bogdanovich T, Clark C, Ednie L, Lin G, Smith K, Shapiro S, et al. Activities of ceftobiprole, a novel broad-spectrum cephalosporin, against Haemophilus influenzae and Moraxella catarrhalis. Antimicrob Agents Chemother.

American Society for Microbiology Journals. 2006;50:2050-7.
82. Marti S, Sánchez-Céspedes J, Espinal P, Vila J. In vitro activity of ceftobiprole against Acinetobacter baumannii clinical isolates. Int J Antimicrob Agents.

Elsevier. 2009;34:265-7.

83. Barbour A, Schmidt S, Sabarinath SN, Grant M, Seubert $\mathrm{C}$, Skee D, et al. Soft-tissue penetration of ceftobiprole in healthy volunteers determined by in vivo microdialysis. Antimicrob Agents Chemother. American Society for Microbiology (ASM). 2009;53:2773-6.

84. Barnea Y, Navon-Venezia S, Kuzmenko B, Artzi N, Carmeli Y. Ceftobiprole medocaril is an effective treatment against methicillin-resistant Staphylococcus aureus (MRSA) mediastinitis in a rat model. Eur J Clin Microbiol Infect Dis.

Springer Berlin. 2014;33:325-9.

85. Noel GJ, Strauss RS, Amsler K, Heep M, Pypstra R, Solomkin JS. Results of a double-blind, randomized trial of ceftobiprole treatment of complicated skin and skin structure infections caused by gram-positive bacteria. Antimicrob Agents Chemother. American Society for Microbiology (ASM). 2008;52:37-44.

86. Chandorkar G, Xiao A, Mouksassi M-S, Hershberger E, Krishna G. Population pharmacokinetics of ceftolozane/tazobactam in healthy volunteers, subjects with varying degrees of renal function and patients with bacterial infections. J Clin Pharmacol.

Wiley. 2015;55:230-9.

87. Aiudi A, Miller B, Krishna G, Adedoyin A, Xiao A. Pharmacokinetics, safety, and tolerability of ceftolozane/tazobactam in healthy Japanese, Chinese, and white subjects. Fundam Clin Pharmacol. 2016;30:625-33.

88. Wooley M, Miller B, Krishna G, Hershberger E, Chandorkar G. Impact of renal function on the pharmacokinetics and safety of ceftolozane-tazobactam. Antimicrob Agents Chemother.

American Society for Microbiology Journals. 2014;58:224955.

89. Monogue ML, Pettit RS, Muhlebach M, Cies JJ, Nicolau DP, Kuti JL. Population pharmacokinetics and safety of ceftolozane-tazobactam in adult cystic fibrosis patients admitted with acute pulmonary exacerbation. Antimicrob Agents Chemother. 2016;60:6578-84.

90. Díaz-Cañestro M, Periañez L, Mulet X. Martin-Pena \& ML, Fraile-Ribot PA, Ayestarán I, et al. Ceftolozane/ tazobactam for the treatment of multidrug resistant Pseudomonas aeruginosa: experience from the Balearic Islands. Eur J Clin Microbiol Infect Dis. 2018;37:2191200.

91. Escolà-Vergé L, Pigrau C, Los-Arcos I, Arévalo Á, Viñado $\mathrm{B}$, Campany D, et al. Ceftolozane/tazobactam for the treatment of XDR Pseudomonas aeruginosa infections. Infection.

Springer Berlin. 2018;46:461-8.

92. Sader HS, Huband MD, Castanheira M, Flamm RK. Pseudomonas aeruginosa antimicrobial susceptibility results from four years (2012 to 2015) of the International Network for Optimal Resistance Monitoring 
Program in the United States. Antimicrob Agents Chemother.

American Society for Microbiology Journals. 2017;61:e0225216.

93. Cabot G, Bruchmann S, Mulet X, Zamorano L, Moyà B, Juan $\mathrm{C}$, et al. Pseudomonas aeruginosa ceftolozanetazobactam resistance development requires multiple mutations leading to overexpression and structural modification of AmpC. Antimicrob Agents Chemother. American Society for Microbiology (ASM). 2014;58:3091-9.

94. Kocsis B, Domokos J, Szabo D. Chemical structure and pharmacokinetics of novel quinolone agents represented by avarofloxacin, delafloxacin, finafloxacin, zabofloxacin and nemonoxacin. Ann Clin Microbiol Antimicrob.

BioMed Central. 2016;15:34.

95. So W, Crandon JL, Nicolau DP. Effects of urine matrix and $\mathrm{pH}$ on the potency of delafloxacin and ciprofloxacin against urogenic Escherichia coli and Klebsiella pneumoniae. J Urol. 2015;194:563-70.

96. Sillerström E, Wahlund E, Nord CE. In vitro activity of ABT-492 against anaerobic bacteria. J Chemother.

Taylor \& Francis. 2004;16:227-9.

97. Firsov AA, Alferova IV, Smirnova MV, Lubenko IY, Portnoy YA, Zinner SH. Comparative pharmacodynamics of the new fluoroquinolone ABT492 and levofloxacin with Streptococcus pneumoniae in an in vitro dynamic model. Int J Antimicrob Agents.

Elsevier. 2005;25:409-13.

98. Hoover R, Marbury TC, Preston RA, Quintas M, Lawrence LE, Paulson SK, et al. Clinical pharmacology of delafloxacin in patients with hepatic impairment. J Clin Pharmacol. 2017;57:328-35.

99. Kocsis B, Szabo D. New treatment options for lower respiratory tract infections. Expert Opin Pharmacother.

Taylor \& Francis. 2017;18:1345-55.

100. Paulson SK, Wood-Horrall RN, Hoover R, Quintas M, Lawrence LE, Cammarata SK. The pharmacokinetics of the CYP3A substrate midazolam after steady-state dosing of delafloxacin. Clin Ther.

Elsevier. 2017;39:1182-90.

101. Litwin JS, Benedict MS, Thorn MD, Lawrence LE, Cammarata SK, Sun E. A thorough QT study to evaluate the effects of therapeutic and supratherapeutic doses of delafloxacin on cardiac repolarization. Antimicrob Agents Chemother.

American Society for Microbiology Journals. 2015;59:346973.

102. Dawe RS, Ferguson J, Ibbotson S, Lawrence L, Paulson S, Duffy E, et al. Lack of phototoxicity potential with delafloxacin in healthy male and female subjects: comparison to lomefloxacin. Photochem Photobiol Sci.

The Royal Society of Chemistry. 2018;17:773-80.

103. Goldstein EJC, Citron DM, Tyrrell KL. In vitro activity of eravacycline and comparator antimicrobials against 143 recent strains of Bacteroides and Parabacteroides species. Anaerobe.

Academic. 2018;52:122-4.
104. Abdallah M, Olafisoye O, Cortes C, Urban C, Landman D, Quale J. Activity of eravacycline against Enterobacteriaceae and Acinetobacter baumannii, including multidrug-resistant isolates, from New York City. Antimicrob Agents Chemother. 2015;59:1802-5.

105. Zhang Y, Lin X, Bush K. In vitro susceptibility of $\beta$ lactamase-producing carbapenem-resistant Enterobacteriaceae (CRE) to eravacycline. J Antibiot (Tokyo).

Nature Publishing Group. 2016;69:600-4.

106. Wright H, Bonomo RA, Paterson DL. New agents for the treatment of infections with Gram-negative bacteria: restoring the miracle or false dawn? Clin Microbiol Infect.

Elsevier. 2017;23:704-12.

107. Newman JV, Zhou J, Izmailyan S, Tsai L. Randomized, double-blind, placebo-controlled studies of the safety and pharmacokinetics of single and multiple ascending doses of eravacycline. Antimicrob Agents Chemother.

American Society for Microbiology Journals. 2018;62:e0117418.

108. Connors KP, Housman ST, Pope JS, Russomanno J, Salerno E, Shore E, et al. Phase I, open-label, safety and pharmacokinetic study to assess bronchopulmonary disposition of intravenous eravacycline in healthy men and women. Antimicrob Agents Chemother.

American Society for Microbiology Journals. 2014;58:2113-8.

109. Tetraphase announces top-line results from IGNITE2 phase 3 clinical trial of eravacycline in cUTI |

Tetraphase Pharmaceuticals [Internet]. [cited 2019 Feb 21]. Available from: https://ir.tphase.com/newsreleases/news-release-details/tetraphase-announcestop-line-results-ignite2-phase-3-clinical

110. Livermore DM, Warner M, Mushtaq S. Activity of MK7655 combined with imipenem against Enterobacteriaceae and Pseudomonas aeruginosa. J Antimicrob Chemother. 2013;68:2286-90.

111. Lapuebla A, Abdallah M, Olafisoye O, Cortes C, Urban C, Landman D, et al. Activity of imipenem with relebactam against gram-negative pathogens from New York City. Antimicrob Agents Chemother.

American Society for Microbiology Journals. 2015;59:502931.

112. Veve MP, Wagner JL. Lefamulin: review of a promising novel pleuromutilin antibiotic. Pharmacother. 2018;38:935-46.

113. Zeitlinger M, Schwameis R, Burian A, Burian B, Matzneller P, Müller M, et al. Simultaneous assessment of the pharmacokinetics of a pleuromutilin, lefamulin, in plasma, soft tissues and pulmonary epithelial lining fluid. J Antimicrob Chemother.

Oxford University Press. 2016;71:1022-6.

114. Mendes RE, Farrell DJ, Flamm RK, Talbot GH, IvezicSchoenfeld Z, Paukner S, et al. In vitro activity of lefamulin tested against Streptococcus pneumoniae with defined serotypes, including multidrug-resistant isolates causing lower respiratory tract infections in 
the United States. Antimicrob Agents Chemother. 2016;60:4407-11.

115. Jacobsson S, Paukner S, Golparian D, Jensen IS, Unemo $M$. In vitro activity of the novel pleuromutilin lefamulin (BC-3781) and effect of efflux pump inactivation on multidrug-resistant and extensively drug-resistant Neisseria gonorrhoeae. Antimicrob Agents Chemother. American Society for Microbiology Journals. 2017;61:e0149717.

116. Prince WT, Ivezic-Schoenfeld Z, Lell C, Tack KJ, Novak R, Obermayr F, et al. Phase II clinical study of BC3781, a pleuromutilin antibiotic, in treatment of patients with acute bacterial skin and skin structure infections. Antimicrob Agents Chemother. 2013;57:2087-94.

117. Alexander E, Goldberg L, Das A, Moran GJ, Sandrock C, Gasink LB, et al. LB6. Oral lefamulin is safe and effective in the treatment of adults with CommunityAcquired Bacterial Pneumonia (CABP): results of Lefamulin Evaluation Against Pneumonia (LEAP 2) study. Open Forum Infect Dis.

Oxford University Press. 2018;5:S761.

118. Rubino CM, Bhavnani SM, Loutit JS, Morgan EE, White D, Dudley MN, et al. Phase 1 study of the safety, tolerability, and pharmacokinetics of vaborbactam and meropenem alone and in combination following single and multiple doses in healthy adult subjects. Antimicrob Agents Chemother.

American Society for Microbiology Journals. 2018;62:e0222817.

119. Griffith DC, Loutit JS, Morgan EE, Durso S, Dudley $\mathrm{MN}$. Phase 1 study of the safety, tolerability, and pharmacokinetics of the $\beta$-lactamase inhibitor vaborbactam (RPX7009) in healthy adult subjects. Antimicrob Agents Chemother.

American Society for Microbiology Journals. 2016;60:632632.

120. Cass RT, Brooks CD, Havrilla NA, Tack KJ, Borin MT, Young D, et al. Pharmacokinetics and safety of single and multiple doses of ACHN-490 injection administered intravenously in healthy subjects. Antimicrob Agents Chemother.

American Society for Microbiology Journals. 2011;55:587480.

121. Castanheira M, Davis AP, Mendes RE, Serio AW, Krause KM, Flamm RK. In vitro activity of plazomicin against gram-negative and gram-positive isolates collected from U.S. hospitals and comparative activities of aminoglycosides against carbapenem-resistant Enterobacteriaceae and isolates carrying carbapenemase genes. Antimicrob Agents Chemother.

American Society for Microbiology Journals. 2018;62:e003138.

122. Castanheira M, Deshpande LM, Woosley LN, Serio AW, Krause KM, Flamm RK. Activity of plazomicin compared with other aminoglycosides against isolates from European and adjacent countries, including Enterobacteriaceae molecularly characterized for aminoglycoside-modifying enzymes and other resistance mechanisms. J Antimicrob Chemother. 2018;73(12):3346-54.

123. Olsen SC, Carlson SA. In vitro bactericidal activity of aminoglycosides, including the next-generation drug plazomicin, against Brucella spp. Int J Antimicrob Agents.

Elsevier. 2015;45:76-8.

124. Jamieson BD, Ciric S, Fernandes P. Safety and pharmacokinetics of solithromycin in subjects with hepatic impairment. Antimicrob Agents Chemother. American Society for Microbiology (ASM). 2015;59:4379-86.

125. Gonzalez D, Palazzi DL, Bhattacharya-Mithal L, AlUzri A, James LP, Bradley J, et al. Solithromycin pharmacokinetics in plasma and dried blood spots and safety in adolescents. Antimicrob Agents Chemother. American Society for Microbiology Journals. 2016;60:2572-6. 126. Darpo B, Sager PT, Fernandes P, Jamieson BD, Keedy $\mathrm{K}$, Zhou M, et al. Solithromycin, a novel macrolide, does not prolong cardiac repolarization: a randomized, three-way crossover study in healthy subjects. J Antimicrob Chemother.

Oxford University Press. 2017;72:515-21.

127. Rodvold KA, Gotfried MH, Still JG, Clark K, Fernandes P. Comparison of plasma, epithelial lining fluid, and alveolar macrophage concentrations of solithromycin (CEM-101) in healthy adult subjects. Antimicrob Agents Chemother. 2012;56:5076-81.

128. Putnam SD, Castanheira M, Moet GJ, Farrell DJ, Jones RN. CEM-101, a novel fluoroketolide: antimicrobial activity against a diverse collection of Gram-positive and Gram-negative bacteria. Diagn Microbiol Infect Dis.

Elsevier. 2010;66:393-401.

129. Jensen JS, Fernandes P, Unemo M. In vitro activity of the new fluoroketolide solithromycin (CEM-101) against macrolide-resistant and -susceptible Mycoplasma genitalium strains. Antimicrob Agents Chemother;

American Society for Microbiology Journals. 2014;58:3151-6.

130. Mallegol J, Fernandes P, Melano RG, Guyard C. Antimicrobial activity of solithromycin against clinical isolates of Legionella pneumophila serogroup 1. Antimicrob Agents Chemother.

American Society for Microbiology Journals. 2014;58:909-15.

131. Oldach D, Clark K, Schranz J, Das A, Craft JC, Scott D, et al. Randomized, double-blind, multicenter phase 2 study comparing the efficacy and safety of oral solithromycin (CEM-101) to those of oral levofloxacin in the treatment of patients with community-acquired bacterial pneumonia. Antimicrob Agents Chemother.

American Society for Microbiology Journals. 2013;57:252634.

\section{Publisher's Note}

Springer Nature remains neutral with regard to jurisdictional claims in published maps and institutional affiliations. 